\title{
Exceptional Legendre Polynomials and Confluent Darboux Transformations
}

\author{
María Ángeles GARCÍA-FERRERO ${ }^{\dagger^{1}}$, David GÓMEZ-ULLATE ${ }^{\dagger^{2} \dagger^{3}}$ and Robert MILSON ${ }^{\dagger^{4}}$ \\ $\dagger^{1}$ Institut für Angewandte Mathematik, Ruprecht-Karls-Universität Heidelberg, \\ Im Neunheimer Feld 205, 69120 Heidelberg, Germany \\ E-mail: garciaferrero@uni-heidelberg.de \\ $\dagger^{2}$ Departamento de Ingeniería Informática, Escuela Superior de Ingeniería, \\ Universidad de Cádiz, 11519 Puerto Real, Spain \\ E-mail: david.gomezullate@uca.es \\ $\dagger^{3}$ Departamento de Física Teórica, Universidad Complutense de Madrid, 28040 Madrid, Spain \\ $\dagger^{4}$ Department of Mathematics and Statistics, Dalhousie University, \\ Halifax, NS, B3H 3J5, Canada \\ E-mail: rmilson@dal.ca
}

Received September 22, 2020, in final form February 03, 2021; Published online February 20, 2021 https://doi.org/10.3842/SIGMA.2021.016

\begin{abstract}
Exceptional orthogonal polynomials are families of orthogonal polynomials that arise as solutions of Sturm-Liouville eigenvalue problems. They generalize the classical families of Hermite, Laguerre, and Jacobi polynomials by allowing for polynomial sequences that miss a finite number of "exceptional" degrees. In this paper we introduce a new construction of multi-parameter exceptional Legendre polynomials by considering the isospectral deformation of the classical Legendre operator. Using confluent Darboux transformations and a technique from inverse scattering theory, we obtain a fully explicit description of the operators and polynomials in question. The main novelty of the paper is the novel construction that allows for exceptional polynomial families with an arbitrary number of real parameters.
\end{abstract}

Key words: exceptional orthogonal polynomials; Darboux transformations; isospectral deformations

2020 Mathematics Subject Classification: 33C47; 34L10; 34A05

\section{Introduction and main results}

Exceptional orthogonal polynomials (XOPs) are complete families of orthogonal polynomials that arise as eigenfunctions of a Sturm-Liouville eigenvalue problem [11]. XOPs are more general than classical OPs, because the degree sequence of the polynomial family can have a finite number of missing, "exceptional" degrees. As in the classical theory, XOPs fall into three broad classes: Hermite, Laguerre and Jacobi, depending on whether the domain of orthogonality is the full line, the half-line or a finite interval [7]. Unlike the classical case, the corresponding exceptional second-order operator has rational rather than polynomial coefficients.

Exceptional polynomials appear in mathematical physics as bound states of exactly solvable rational extensions $[10,24,26]$ and exact solutions to Dirac's equation [27]. They appear also in connection with super-integrable systems $[21,25]$ and finite-gap potentials [17]. From a mathematical point of view, the main results are concerned with the full classification of exceptional polynomials [7, 12], properties of their zeros [14, 18, 20], and recurrence relations [5, 13, 22, 23].

At the time of this writing, the most general construction of exceptional Jacobi polynomials [3,6] involves a finite number of discrete parameters and is given in terms of a Wronskian- 
like determinant of classical Jacobi polynomials, indexed by two partitions. The purpose of this note is to show that the class of exceptional orthogonal polynomials is much richer than previously thought. We do this by studying the class of exceptional Legendre polynomials, which cannot be obtained using the standard approach of multi-step Darboux transformations indexed by partitions. The main novelty of the new families is that they contain an arbitrary number of continuous deformation parameters. Another innovation is the use of integral rather than differential operators in the construction of the exceptional polynomials.

Definition 1.1. Let $\tau=\tau(z)$ be a polynomial. We say that the operator

$$
\hat{T}(\tau)=\left(1-z^{2}\right)\left(D_{z}^{2}-2 \frac{\tau_{z}}{\tau} D_{z}+\frac{\tau_{z z}}{\tau}\right)-2 z D_{z}
$$

is an exceptional Legendre operator if there exist polynomials $\left\{\hat{P}_{i}(z)\right\}_{i \in \mathbb{N}_{0}}$ and constants $\left\{\lambda_{i}\right\}_{i \in \mathbb{N}_{0}}$, where $\mathbb{N}_{0}=\{0,1, \ldots\}$, such that

$$
\hat{T}(\tau) \hat{P}_{i}=\lambda_{i} \hat{P}_{i}
$$

and such that the degree sequence $\left\{\operatorname{deg} \hat{P}_{i}\right\}_{i \in \mathbb{N}_{0}}$ is missing finitely many "exceptional" degrees [11].

Note that, in making this definition, we are not assuming that $\operatorname{deg} \hat{P}_{i}=i$.

Remark 1.2. As a direct consequence of this definition, if $\tau(z)$ has no zeros on $[-1,1]$ and if the eigenvalues are distinct, then the resulting eigenpolynomials are orthogonal relative to the inner product

$$
\int_{-1}^{1} \frac{\hat{P}_{i_{1}}(z) \hat{P}_{i_{2}}(z)}{\tau(z)^{2}} \mathrm{~d} z=0, \quad i_{1} \neq i_{2}
$$

In this case, the eigenpolynomials $\left\{\hat{P}_{i}(z)\right\}_{i \in \mathbb{N}_{0}}$ may define a complete orthogonal polynomial system, which motivates the following definition.

Definition 1.3. Let $\tau(z)$ be a polynomial that does not vanish in $[-1,1]$. The set $\left\{\hat{P}_{i}(z)\right\}_{i \in \mathbb{N}_{0}}$ is a family of exceptional Legendre polynomials if

(i) $\left\{\hat{P}_{i}(z)\right\}_{i \in \mathbb{N}_{0}}$ are eigenfunctions of a Sturm-Liouville problem in $[-1,1]$.

(ii) $\left\{\operatorname{deg} \hat{P}_{i}\right\}_{i \in \mathbb{N}_{0}}$ contains all but finitely many positive integers.

(iii) The polynomials $\left\{\hat{P}_{i}(z)\right\}_{i \in \mathbb{N}_{0}}$ satisfy the orthogonality relation (1.2).

(iv) The polynomials $\left\{\hat{P}_{i}(z)\right\}_{i \in \mathbb{N}_{0}}$ form a complete set in the Hilbert space $\mathrm{L}^{2}\left([-1,1], \tau^{-2} \mathrm{~d} z\right)$.

In other words, exceptional Legendre polynomials are just exceptional polynomials defined in $[-1,1]$ with orthogonality weight $W(z)=\tau(z)^{-2}$, where $\tau(z)$ is a polynomial not vanishing in $[-1,1]$. It should be noted, however, that the standard construction of exceptional Jacobi polynomials based on a multi-index determinant labelled by two partitions [6, equation (5.1)] does not allow parameters $\alpha=\beta=0$ (see [3, equation (2.36)]). Thus, the construction of exceptional Legendre polynomials requires a different approach, which we present in this paper.

It is known [7, Theorem 1.2] that every exceptional operator can be related to a classical Bochner operator by a finite number of Darboux transformations. This is true in particular for an exceptional operator having the form (1.1), where the degree of $\tau(z)$ is equal to the number of exceptional degrees. The new exceptional polynomial families introduced in this paper do not invalidate the classification result [7, Theorem 1.2], but rather they highlight the fact that the 
full class of Darboux transformations leading to exceptional polynomials is larger than previously thought.

As a matter of fact, we will consider in this paper a new class of exceptional operators that are obtained from the classical Legendre operator

$$
T:=\hat{T}(1)=\left(1-z^{2}\right) D_{z}^{2}-2 z D_{z}
$$

by the application of a finite number of confluent Darboux transformations (CDTs) [16], also known as the "double commutator" method [9]. A CDT applied within a spectral gap of a second-order self-adjoint operator allows to add one eigenvalue to the spectrum. We will relate $T$ to $\hat{T}(\tau)$ by a chain of CDTs, but the commutation procedure we consider is performed at an existing eigenvalue. The resulting spectral transformation for every confluent pair "deletes" an existing eigenvalue and then "adds" it back. ${ }^{1}$ The overall effect is that of an isospectral transformation [19].

An important feature of confluent Darboux transformations is that every confluent pair of transformations naturally introduces an extra deformation parameter. Known instances of exceptional Jacobi polynomials are indexed by discrete parameters and cannot be continuously deformed into their classical counterparts. By contrast, after performing $n$ CDTs on the classical Legendre operator (1.3) at distinct energy levels indexed by $\boldsymbol{m}=\left(m_{1}, \ldots, m_{n}\right) \in \mathbb{N}_{0}^{n}$ we will arrive at an exceptional Legendre operator

$$
T_{\boldsymbol{m}}\left(\boldsymbol{t}_{\boldsymbol{m}}\right)=\hat{T}\left(\tau_{\boldsymbol{m}}\left(z ; t_{\boldsymbol{m}}\right)\right)
$$

that depends on $n$ real parameters $\boldsymbol{t}_{\boldsymbol{m}}=\left(t_{m_{1}}, \ldots, t_{m_{n}}\right) \in \mathbb{R}^{n}$. The polynomial eigenfunctions of $T_{\boldsymbol{m}}\left(\boldsymbol{t}_{\boldsymbol{m}}\right)$ are exceptional Legendre polynomials $\left\{P_{\boldsymbol{m}, i}\left(z ; \boldsymbol{t}_{\boldsymbol{m}}\right)\right\}_{i \in \mathbb{N}_{0}}$, which depend on $n$ real parameters $\boldsymbol{t}_{\boldsymbol{m}}=\left(t_{m_{1}}, \ldots, t_{m_{n}}\right)$, and can be continuously deformed to the classical Legendre polynomials by letting $\boldsymbol{t}_{\boldsymbol{m}} \rightarrow 0$.

Adapting certain methodologies from the theory of inverse scattering [1, 4, 29], we are able to exhibit a determinantal representation of $\tau_{\boldsymbol{m}}\left(z ; \boldsymbol{t}_{\boldsymbol{m}}\right)$ that is formally similar to the construction of KdV multi-solitons. The difference here is that, instead of dressing the zero potential, we isospectrally deform a particular instance of the Darboux-Poschl-Teller potential [15] by modifying the normalizations of a finite number of the corresponding bound states. Another feature of our approach is that, rather than working with a Schrödinger operator, we remain in a polynomial setting by utilizing the gauge and coordinate of the Legendre operator. The result is a constructive procedure that can be easily implemented using a computer algebra system.

\subsection{Notation and definitions}

The base case of the construction is the classical Legendre operator $T$, shown in (1.3), and the classical Legendre polynomials [30]

$$
P_{i}(z):=\frac{2^{-i}}{i !} D_{z}^{i}\left(z^{2}-1\right)^{i}=2^{-i} \sum_{k=0}^{i}\left(\begin{array}{l}
i \\
k
\end{array}\right)^{2}(z-1)^{i-k}(z+1)^{k}, \quad i \in \mathbb{N}_{0} .
$$

These classical orthogonal polynomials do have $\operatorname{deg} P_{i}=i$, they satisfy the eigenvalue relation

$$
T P_{i}=-i(i+1) P_{i}, \quad i \in \mathbb{N}_{0},
$$

and they form an $\mathrm{L}^{2}$-complete orthogonal family relative to the inner product

$$
\int_{-1}^{1} P_{i_{1}}(z) P_{i_{2}}(z) \mathrm{d} z=\frac{2}{2 i_{1}+1} \delta_{i_{1} i_{2}}, \quad i_{1}, i_{2} \in \mathbb{N}_{0} .
$$

\footnotetext{
${ }^{1}$ This is true in a formal sense only, as the intermediate potential is singular.
} 
Before we can state the main results of the paper, we would like to fix some notation conventions to be used throughout the paper. Bold symbols such as $\boldsymbol{m}, \boldsymbol{t}$ or $\boldsymbol{Q}$ will represent tuples of integers, real numbers or polynomials (one dimensional objects), while calligraphic symbols like $\mathcal{R}$ will denote matrices. To access the components of a vector or tensor we will employ square brackets, i.e., $[\mathcal{R}]_{k \ell}$ denotes the $(k, \ell)$ entry of $\mathcal{R}$. In addition, given an $n \times n$ matrix $\mathcal{R}$ and integers $1 \leq k<\ell \leq n$, we denote by $\mathcal{M}_{k, \ell}(\mathcal{R})$ the $(\ell-k+1) \times(\ell-k+1)$ square submatrix of $\mathcal{R}$ that includes the intersection of rows and columns from $k$ to $\ell$.

If $\boldsymbol{m}=\left(m_{1}, \ldots, m_{n}\right)$ is an $n$-tuple and $k \in\{1, \ldots, n\}$, we will denote by $\boldsymbol{m}^{\langle k\rangle}$ the $(n-1)$-tuple where the element $[\boldsymbol{m}]_{k}$ is removed, i.e., $\boldsymbol{m}^{\langle k\rangle}=\left(m_{1}, \ldots, m_{k-1}, m_{k+1}, \ldots, m_{n}\right)$, and by $\boldsymbol{m}_{k}$ the $k$-tuple formed by the first $k$ elements of $\boldsymbol{m}$, i.e., $\boldsymbol{m}_{k}=\left(m_{1}, \ldots, m_{k}\right)$. In particular, we may write explicitly $\boldsymbol{m}_{n}$ instead of $\boldsymbol{m}$ whenever the context requires to emphasize the length of the tuple, mostly in the proofs by induction or recurrence relations.

Associated to an $n$-tuple of integers $\boldsymbol{m}=\left(m_{1}, \ldots, m_{n}\right) \in \mathbb{N}_{0}^{n}$, we will define the $n$-tuple of real parameters $\boldsymbol{t}_{\boldsymbol{m}}=\left(t_{m_{1}}, \ldots, t_{m_{n}}\right) \in \mathbb{R}^{n}$. Semicolons will be used to separate objects of different nature. Commas will be used for tuple concatenation, e.g., if $i_{1}, \ldots, i_{k} \in \mathbb{N}_{0},\left(\boldsymbol{m}, i_{1}, \ldots, i_{k}\right)$ denotes the $(n+k)$-tuple $\left(m_{1}, \ldots, m_{n}, i_{1}, \ldots, i_{k},\right)$. Similarly, we have $\left(\boldsymbol{t}_{\boldsymbol{m}}, t_{i_{1}}, \ldots, t_{i_{k}}\right)=\boldsymbol{t}_{\left(\boldsymbol{m}, i_{1}, \ldots, i_{k}\right)}$. Often, we will omit the parentheses when denoting 1-tuples, e.g., $m_{1}$ instead of $\left(m_{1}\right)$. Finally, we will use, depending on the context, the following notation for derivatives of a function $f$ with respect to $z: D_{z} f, f^{\prime}$ and $f_{z}$.

With this notation in mind, we proceed to define the main objects of this paper.

Definition 1.4. Given an $n$-tuple $\boldsymbol{m} \in \mathbb{N}_{0}^{n}$ and the associated $\boldsymbol{t}_{\boldsymbol{m}} \in \mathbb{R}^{n}$, we define $\mathcal{R}_{\boldsymbol{m}}\left(z ; \boldsymbol{t}_{\boldsymbol{m}}\right)$ as the $n \times n$ matrix with polynomial entries given by

$$
\left[\mathcal{R}_{\boldsymbol{m}}\left(z ; t_{\boldsymbol{m}}\right)\right]_{k \ell}=\delta_{k \ell}+t_{m_{\ell}} R_{m_{k} m_{\ell}}(z), \quad k, \ell \in\{1, \ldots, n\}
$$

where

$$
R_{m_{k} m_{\ell}}(z):=\int_{-1}^{z} P_{m_{k}}(u) P_{m_{\ell}}(u) \mathrm{d} u
$$

and $P_{i}(z)$ denote the classical Legendre polynomials (1.4). We denote its determinant by

$$
\tau_{\boldsymbol{m}}\left(z ; \boldsymbol{t}_{\boldsymbol{m}}\right):=\operatorname{det} \mathcal{R}_{\boldsymbol{m}}\left(z ; \boldsymbol{t}_{\boldsymbol{m}}\right)
$$

We define the $n$-tuple of polynomials

$$
\boldsymbol{Q}_{\boldsymbol{m}}^{\mathrm{T}}\left(z ; \boldsymbol{t}_{\boldsymbol{m}}\right):=\tau_{\boldsymbol{m}}\left(z ; \boldsymbol{t}_{\boldsymbol{m}}\right) \mathcal{R}_{\boldsymbol{m}}\left(z ; \boldsymbol{t}_{\boldsymbol{m}}\right)^{-1}\left(P_{m_{1}}(z), \ldots, P_{m_{n}}(z)\right)^{\mathrm{T}}
$$

Finally, for $i \in \mathbb{N}_{0}$, we define the polynomials

$$
P_{\boldsymbol{m} ; i}\left(z ; \boldsymbol{t}_{\boldsymbol{m}}\right):=\left[\boldsymbol{Q}_{(\boldsymbol{m}, i)}\left(z ; \boldsymbol{t}_{(\boldsymbol{m}, i)}\right)\right]_{n+1} .
$$

Note that, by construction, $\tau_{\boldsymbol{m}}\left(z ; \boldsymbol{t}_{\boldsymbol{m}}\right)$ is symmetric in $\boldsymbol{m}$ and $\boldsymbol{Q}_{\boldsymbol{m}}\left(z ; \boldsymbol{t}_{\boldsymbol{m}}\right)$ is equivariant with respect to permutations of $\boldsymbol{m}$. In addition, $P_{\boldsymbol{m} ; i}\left(z ; \boldsymbol{t}_{\boldsymbol{m}}\right)$ is symmetric in $\boldsymbol{m}$ and does not depend on $t_{i}$ since $\tau_{(\boldsymbol{m}, i)}\left(z ; \boldsymbol{t}_{(\boldsymbol{m}, i)}\right)\left[\mathcal{R}_{(\boldsymbol{m}, i)}\left(z ; \boldsymbol{t}_{(\boldsymbol{m}, i)}\right)^{-1}\right]_{n+1, j}$ correspond to the minors of the last column of $\mathcal{R}_{(\boldsymbol{m}, i)}\left(z ; \boldsymbol{t}_{(\boldsymbol{m} ; i)}\right)$, the only column where $t_{i}$ appears. 
For example, for $m_{1}, m_{2} \in \mathbb{N}_{0}$ we have

$$
\begin{aligned}
& \tau_{m_{1}}\left(z ; t_{m_{1}}\right)=1+t_{m_{1}} R_{m_{1} m_{1}}(z), \\
& \mathcal{R}_{\left(m_{1}, m_{2}\right)}\left(z ; \boldsymbol{t}_{\left(m_{1}, m_{2}\right)}\right)=\left(\begin{array}{cc}
1+t_{m_{1}} R_{m_{1} m_{1}}(z) & t_{m_{2}} R_{m_{1} m_{2}}(z) \\
t_{m_{1}} R_{m_{2} m_{1}}(z) & 1+t_{m_{2}} R_{m_{2} m_{2}}(z)
\end{array}\right), \\
& \tau_{\left(m_{1}, m_{2}\right)}\left(z ; \boldsymbol{t}_{\left(m_{1}, m_{2}\right)}\right)= 1+t_{m_{1}} R_{m_{1} m_{1}}(z)+t_{m_{2}} R_{m_{2} m_{2}}(z) \\
&+t_{m_{1}} t_{m_{2}}\left(R_{m_{1} m_{1}}(z) R_{m_{2} m_{2}}(z)-R_{m_{1} m_{2}}^{2}(z)\right), \\
& \boldsymbol{Q}_{\left(m_{1}, m_{2}\right)}\left(z ; \boldsymbol{t}_{\left(m_{1}, m_{2}\right)}\right)=\left(\begin{array}{c}
\left(1+t_{m_{2}} R_{m_{2} m_{2}}(z)\right) P_{m_{1}}(z)-t_{m_{2}} R_{m_{1} m_{2}}(z) P_{m_{2}}(z) \\
\left(1+t_{m_{1}} R_{m_{1} m_{1}}(z)\right) P_{m_{2}}(z)-t_{m_{1}} R_{m_{2} m_{1}}(z) P_{m_{1}}(z)
\end{array}\right), \\
& P_{m_{1} ; i}\left(z ; t_{m_{1}}\right)=\left(1+t_{m_{1}} R_{m_{1} m_{1}}(z)\right) P_{i}(z)-t_{m_{1}} R_{i m_{1}}(z) P_{m_{1}}(z), \quad i \in \mathbb{N}_{0} .
\end{aligned}
$$

After defining these objects, we are now ready to state the results.

\subsection{Main results}

The main result of this paper states that the polynomials $\left\{P_{\boldsymbol{m} ; i}\left(z ; \boldsymbol{t}_{\boldsymbol{m}}\right)\right\}_{i \in \mathbb{N}_{0}}$ defined by (1.5)-(1.8) are exceptional Legendre polynomials, provided the real parameters $\boldsymbol{t}_{\boldsymbol{m}}$ satisfy certain constraints to ensure that $\tau_{\boldsymbol{m}}\left(z ; t_{\boldsymbol{m}}\right)$ has constant sign on $z \in[-1,1]$.

Theorem 1.5. For $\boldsymbol{m} \in \mathbb{N}_{0}^{n}$, consider the operator

$$
T_{\boldsymbol{m}}\left(\boldsymbol{t}_{\boldsymbol{m}}\right):=\hat{T}\left(\tau_{\boldsymbol{m}}\left(z ; \boldsymbol{t}_{\boldsymbol{m}}\right)\right),
$$

given by (1.1) and (1.6). Then $T_{\boldsymbol{m}}\left(t_{\boldsymbol{m}}\right)$ is an exceptional Legendre operator that satisfies

$$
T_{\boldsymbol{m}}\left(\boldsymbol{t}_{\boldsymbol{m}}\right) P_{\boldsymbol{m} ; i}\left(z ; \boldsymbol{t}_{\boldsymbol{m}}\right)=-i(i+1) P_{\boldsymbol{m} ; i}\left(z ; \boldsymbol{t}_{\boldsymbol{m}}\right), \quad i \in \mathbb{N}_{0}
$$

with $P_{\boldsymbol{m} ; i}\left(z ; \boldsymbol{t}_{\boldsymbol{m}}\right)$ as in $(1.8)$.

In light of (1.9), we may refer to $P_{\boldsymbol{m} ; i}\left(z ; \boldsymbol{t}_{\boldsymbol{m}}\right)$, where $i \in \mathbb{N}_{0}$ varies and $\boldsymbol{m}$ and $\boldsymbol{t}_{\boldsymbol{m}}$ are fixed, as exceptional Legendre polynomials. This requires according to Remark 1.2 and Definition 1.3 that $\tau_{\boldsymbol{m}}\left(z ; t_{\boldsymbol{m}}\right)$ does not vanish on $[-1,1]$. The following theorem gives necessary and sufficient conditions for this to be true. In that case, like their classical counterparts, the polynomials $\left\{P_{\boldsymbol{m} ; i}\left(z ; \boldsymbol{t}_{\boldsymbol{m}}\right)\right\}_{i \in \mathbb{N}_{0}}$ are orthogonal and complete.

Theorem 1.6. For $\boldsymbol{m} \in \mathbb{N}_{0}^{n}$ with $m_{1}, \ldots, m_{n}$ distinct, the polynomial $\tau_{\boldsymbol{m}}\left(z ; \boldsymbol{t}_{\boldsymbol{m}}\right)$ in (1.6) has no zeros on $[-1,1]$ if and only if

$$
t_{m_{j}}>-m_{j}-\frac{1}{2}, \quad j \in\{1, \ldots, n\} .
$$

If the above conditions hold, then $\left\{P_{\boldsymbol{m} ; i}\left(z ; \boldsymbol{t}_{\boldsymbol{m}}\right)\right\}_{i \in \mathbb{N}_{0}}$ are exceptional Legendre polynomials with

$$
\int_{-1}^{1} \frac{P_{\boldsymbol{m} ; i_{1}}\left(u ; \boldsymbol{t}_{\boldsymbol{m}}\right) P_{\boldsymbol{m} ; i_{2}}\left(u ; \boldsymbol{t}_{\boldsymbol{m}}\right)}{\tau_{\boldsymbol{m}}\left(z ; \boldsymbol{t}_{\boldsymbol{m}}\right)^{2}} \mathrm{~d} u=\frac{2}{1+2 i_{1}+2\left(\delta_{i_{1} m_{1}}+\cdots+\delta_{i_{1} m_{n}}\right) t_{i_{1}}} \delta_{i_{1} i_{2}}, \quad i_{1}, i_{2} \in \mathbb{N}_{0} .
$$

and $\mathrm{L}^{2}$-completeness in $[-1,1]$ relative to the measure $\tau_{\boldsymbol{m}}\left(z ; \boldsymbol{t}_{\boldsymbol{m}}\right)^{-2} \mathrm{~d} z$.

Remark 1.7. Note that we could reformulate the above result without the assumption that $m_{1}, \ldots, m_{n}$ are distinct. However, there is no extra benefit in doing this, as demonstrated by Proposition 3.6 below. Assuming that the indices $m_{1}, \ldots, m_{n}$ are all distinct does not entail any loss of generality. 
The rest of the paper is organized as follows: in Section 2 we study exceptional Legendre operators connected by a single step rational confluent Darboux transformation, which involves in fact two Darboux transformations at the same factorization energy. We will iterate these results in Section 3 to consider any number of confluent Darboux transformations and we will relate this construction with the objects in Definition 1.4, thus yielding the proofs of the main theorems. Finally, in Section 4 we give some explicit examples of the new exceptional Legendre families.

\section{One step confluent Darboux transformations}

In this section we collect a number of relevant Propositions for the proofs of the Theorems 1.5 and 1.6. We introduce the concept of a rational confluent Darboux transformation and we show that this transformation preserves the class of exceptional Legendre operators.

Before introducing rational confluent Darboux transformation, we recall 2-step ordinary Darboux transformations between two operators $T_{1}$ and $T_{2}$ with rational coefficients. If $A_{1}$, $A_{2}, B_{1}, B_{2}$ are first-order differential operators with rational coefficients, and $\lambda_{1}, \lambda_{2}$ two constants, consider the following 2-step rational Darboux transformation:

$$
\begin{aligned}
& T_{1}=B_{1} A_{1}+\lambda_{1}, \\
& \tilde{T}=A_{1} B_{1}+\lambda_{1}=A_{2} B_{2}+\lambda_{2}, \\
& T_{2}=B_{2} A_{2}+\lambda_{2} .
\end{aligned}
$$

The first transformation at energy level $\lambda_{1}$ maps $T_{1}$ to $\tilde{T}$ and is state-deleting, while the second transformation at energy level $\lambda_{2}$ maps $\tilde{T}$ to $T_{2}$ and is state-adding (or equivalently, the inverse transformation from $T_{2}$ to $\tilde{T}$ is state-deleting). The confluent version arises when $\lambda_{1}=\lambda_{2}$, i.e., we use seed functions at each of the two steps which are (at least formally) eigenfunctions of the corresponding (formal) operator with the same eigenvalue. A full discussion of confluent Darboux transformations from this point of view can be seen, for instance, in [28]. We can make this notion more precise in the following definition.

Definition 2.1. Let $T_{1}, T_{2}$ be second-order operators with rational coefficients. We will say that $T_{1}$ and $T_{2}$ are related by a rational confluent Darboux transformation if there exist firstorder operators $A_{1}, A_{2}, B_{1}, B_{2}$, all with rational coefficients, and a constant $\lambda$ such that

$$
A_{1} B_{1}=A_{2} B_{2}, \quad T_{1}=B_{1} A_{1}+\lambda, \quad T_{2}=B_{2} A_{2}+\lambda .
$$

Given polynomials $\tau(z), \phi(z)$, we define the rational operators

$$
\begin{aligned}
& A(\tau, \phi):=\tau^{-1}\left(\phi D_{z}-\phi_{z}\right), \\
& B(\phi, \tau):=A(\phi, \tau) \circ\left(1-z^{2}\right)=\phi^{-1}\left(\left(1-z^{2}\right)\left(\tau D_{z}-\tau_{z}\right)-2 z \tau\right) .
\end{aligned}
$$

The form of these operators coincides with the general form of the first-order operators appearing in factorization of operators given in [7, Proposition 3.5], with a particular choice that ensures that operator $\hat{T}(\tau)=B(\phi, \tau) A(\tau, \phi)$ is in the natural gauge [7, Definition 5.1]. In the proofs, we will use the fact that, for a given function $f$, we have

$$
A(\tau, \phi) f=\tau^{-1} \operatorname{Wr}(\phi, f),
$$

where Wr denotes the Wronskian determinant.

Throughout this section, we consider an exceptional Legendre operator $\hat{T}(\tau)$ with polynomials $\left\{\pi_{i}(z)\right\}_{i \in \mathbb{N}_{0}}$ that satisfy

$$
\hat{T}(\tau) \pi_{i}=\lambda_{i} \pi_{i}, \quad \lambda_{i_{1}} \neq \lambda_{i_{2}} \quad \text { if } \quad i_{1} \neq i_{2}, \quad i, i_{1}, i_{2} \in \mathbb{N}_{0} .
$$


Our goal is to apply a rational CDT on this operator. To this end, for $m \in \mathbb{N}_{0}$ and $t \in \mathbb{R}$, let us define the following objects:

$$
\begin{aligned}
& \rho_{i_{1} i_{2}}(z):=\int_{-1}^{z} \frac{\pi_{i_{1}}(u) \pi_{i_{2}}(u)}{\tau(u)^{2}} \mathrm{~d} u, \quad i_{1}, i_{2} \in \mathbb{N}_{0}, \\
& \tau_{m}(z ; t):=\tau(z)\left(1+t \rho_{m m}(z)\right), \\
& \pi_{m ; i}(z ; t):=\left(1+t \rho_{m m}(z)\right) \pi_{i}(z)-t \rho_{i m}(z) \pi_{m}(z), \quad i \in \mathbb{N}_{0} .
\end{aligned}
$$

For a lighter notation, we may omit the $t$ dependence and write $\tau_{m}$ instead of $\tau_{m}(z ; t)$. Note that $\tau$ might already depend on a number of real parameters, so $\tau_{m}$ will depend on the same parameters as $\tau$, plus an extra parameter $t$.

Remark 2.2. In the rest of this Section, i.e., for the following four Propositions, we shall assume that $\rho_{i_{1} i_{2}}(z)$ defined by $(2.3)$ is a rational function that vanishes at $z=-1$ and $\tau_{m}(z)$ and $\pi_{m ; i}(z, t)$ defined by $(2.4)-(2.5)$ are polynomials in $z$.

If we start from an exceptional Legendre operator (1.1) for a given $\tau$ polynomial with eigenpolynomials $\pi_{i}$, these assumptions are far from obvious by looking at (2.3)-(2.5). In the next section we will see that the assumptions hold whenever (2.2) does, i.e., that the rational CDT between exceptional Legendre families is well defined.

Proposition 2.3. For $m \in \mathbb{N}_{0}$, let $\rho_{m m}(z)$ and $\tau_{m}(z)$ be defined by (2.3)-(2.4) and satisfy the assumptions of Remark 2.2. Then, $\hat{T}(\tau)$ and $\hat{T}\left(\tau_{m}\right)$ are related by a rational confluent Darboux transformation with

$$
\begin{aligned}
& A\left(\tau, \pi_{m}\right) B\left(\pi_{m}, \tau\right)=A\left(\tau_{m}, \pi_{m}\right) B\left(\pi_{m}, \tau_{m}\right), \\
& \hat{T}(\tau)=B\left(\pi_{m}, \tau\right) A\left(\tau, \pi_{m}\right)+\lambda_{m}, \\
& \hat{T}\left(\tau_{m}\right)=B\left(\pi_{m}, \tau_{m}\right) A\left(\tau_{m}, \pi_{m}\right)+\lambda_{m} .
\end{aligned}
$$

Proof. The results follow from direct calculation with the previous definitions.

The following lemma examines the behaviour at the endpoint $z=-1$ of a combination of these objects, and it will be necessary to prove some of the following propositions.

Lemma 2.4. Let $\left\{\pi_{i}\right\}_{i \in \mathbb{N}_{0}}$ be polynomials that satisfy the eigenvalue equation (2.2) with (1.1) and let $\rho_{i_{1}, i_{2}}(z)$ be the rational functions defined by (2.3) that vanish at $z=-1$. Then,

$$
\left.\left(1-z^{2}\right) \frac{\operatorname{Wr}\left(\pi_{i}, \pi_{m}\right)}{\tau^{2}}\right|_{z=-1}=0
$$

Proof. Since $\rho_{i m}(z)$ is rational and vanishes at $z=-1$, we can write for given $\alpha, \beta \in \mathbb{N}_{0}$

$$
\begin{aligned}
& \rho_{i m}(z)=(1+z)^{1+\alpha} q(z), \quad q(-1)=C_{q} \neq 0, \quad \alpha>0, \\
& \tau(z)=(1+z)^{\beta} p(z), \quad p(-1)=C_{p} \neq 0,
\end{aligned}
$$

where $q$ is a rational function and $p$ is a polynomial. Thus

$$
\rho_{i m}^{\prime}(-1)=\left.\frac{\pi_{i} \pi_{m}}{\tau^{2}}\right|_{z=-1}= \begin{cases}C_{q} & \text { if } \alpha=1 \\ 0 & \text { if } \alpha \geq 2\end{cases}
$$

Using the eigenvalue equation we have

$$
\left(1-z^{2}\right)\left(\pi_{i}^{\prime \prime} \pi_{m}-\pi_{i} \pi_{m}^{\prime \prime}\right)-2\left(\left(1-z^{2}\right) \frac{\tau^{\prime}}{\tau}+z\right)\left(\pi_{i}^{\prime} \pi_{m}-\pi_{i} \pi_{m}^{\prime}\right)=\left(\lambda_{i}-\lambda_{m}\right) \pi_{i} \pi_{m} .
$$


Evaluating both sides at $z=-1$, we have

$$
\left.\frac{\mathrm{Wr}\left(\pi_{i}, \pi_{m}\right)}{\tau^{2}}\right|_{z=-1}=\left.\frac{\left(\lambda_{i}-\lambda_{m}\right)}{2(1-2 \beta)} \frac{\pi_{i} \pi_{m}}{\tau^{2}}\right|_{z=-1}<\infty .
$$

Since the previous expression is bounded, the desired result is proved.

The next proposition shows how to build the eigenpolynomials of the transformed operator, by the use of the second order intertwining relations for CDTs

$$
\left(B_{2} A_{1}\right) T_{1}=T_{2}\left(B_{2} A_{1}\right), \quad T_{1}\left(B_{1} A_{2}\right)=\left(B_{1} A_{2}\right) T_{2} .
$$

Proposition 2.5. For $m \in \mathbb{N}_{0}$, let $\rho_{i m}(z), \tau_{m}(z)$ and $\pi_{m ; i}(z, t)$ be defined by $(2.3)-(2.5)$ and satisfy the assumptions of Remark 2.2 .

Then, for $i \in \mathbb{N}_{0}$, we have

$$
\begin{aligned}
& \left(\lambda_{m}-\lambda_{i}\right) \rho_{i m}=\left(1-z^{2}\right) \tau^{-1} A\left(\tau, \pi_{m}\right) \pi_{i}, \\
& \left(\lambda_{m}-\lambda_{i}\right) \pi_{m ; i}=B\left(\pi_{m}, \tau_{m}\right) A\left(\tau, \pi_{m}\right) \pi_{i}, \\
& \hat{T}\left(\tau_{m}\right) \pi_{m ; i}=\lambda_{i} \pi_{m ; i} .
\end{aligned}
$$

Proof. We start by noticing that

$$
\left(\lambda_{m}-\lambda_{i}\right) \rho_{i m}^{\prime}=\left(\lambda_{m}-\lambda_{i}\right) \frac{\pi_{i} \pi_{m}}{\tau^{2}}=\left(\left(1-z^{2}\right) \tau^{-1} A\left(\tau, \pi_{m}\right) \pi_{i}\right)^{\prime},
$$

where for the last equality we use the eigenvalue equation (2.2), or equivalently the SturmLiouville equation

$$
\left(\left(1-z^{2}\right) \tau^{-2} \pi_{i}^{\prime}\right)^{\prime}+\left(1-z^{2}\right) \tau_{z z} \tau^{-3} \pi_{i}=\lambda_{i} \tau^{-2} \pi_{i} .
$$

The first result follows by integration since Lemma 2.4 ensures that

$$
\left.\left(1-z^{2}\right) \tau^{-1} A\left(\tau, \pi_{m}\right) \pi_{i}\right|_{z=-1}=\left.\left(1-z^{2}\right) \frac{\mathrm{Wr}\left(\pi_{m}, \pi_{i}\right)}{\tau^{2}}\right|_{z=-1}=0
$$

The second identity follows by direct calculation using the definitions and previous identities. Indeed, using (2.1) and (2.6) we have

$$
\begin{aligned}
B\left(\pi_{m}, \tau_{m}\right) A\left(\tau, \pi_{m}\right) \pi_{i} & =A\left(\pi_{m}, \tau_{m}\right) \circ\left(1-z^{2}\right)\left(\frac{\left(\lambda_{m}-\lambda_{i}\right) \rho_{i m}}{\left(1-z^{2}\right) \tau^{-1}}\right) \\
& =\left(\lambda_{m}-\lambda_{i}\right) \pi_{m}^{-1} \operatorname{Wr}\left(\tau_{m}, \tau \rho_{i m}\right) \\
& =\left(\lambda_{m}-\lambda_{i}\right) \pi_{m}^{-1} \tau^{2} \operatorname{Wr}\left(1+t \rho_{m m}, \rho_{i m}\right)
\end{aligned}
$$

and deriving $\rho_{i m}$ in (2.3) and using (2.5) leads to the desired result (2.7).

The third identity (2.8) follows trivially from (2.7) and the intertwining relation

$$
\hat{T}\left(\tau_{m}\right) B\left(\pi_{m}, \tau_{m}\right) A\left(\tau, \pi_{m}\right)=B\left(\pi_{m}, \tau_{m}\right) A\left(\tau, \pi_{m}\right) \hat{T}(\tau) .
$$

The next result derives the transformation rule for $\rho_{i_{1} i_{2}}$ under a CDT, and it is key to obtain the norming constants of the transformed polynomials in Proposition 2.7.

Proposition 2.6. For $m, i_{1}, i_{2} \in \mathbb{N}_{0}$, let $\rho_{i_{1} i_{2}}(z), \tau_{m}(z)$ and $\pi_{m ; i}(z, t)$ be defined by $(2.3)-(2.5)$ and satisfy the assumptions of Remark 2.2. Then,

$$
\int_{-1}^{z} \frac{\pi_{m ; i_{1}}(u ; t) \pi_{m ; i_{2}}(u ; t)}{\tau_{m}(u)^{2}} \mathrm{~d} u=\rho_{i_{1} i_{2}}(z)-\frac{t \rho_{i_{1} m}(z) \rho_{i_{2} m}(z)}{1+t \rho_{m m}(z)}, \quad i_{1}, i_{2} \in \mathbb{N}_{0} .
$$


Proof. The identity between the derivatives of both sides can be easily proved by direct computation using the definitions. The desired result follows then by integration since $\rho_{i_{1} i_{2}}(-1)=0$.

The last proposition of this section shows that the CDT of an exceptional Legendre family falls into the same class under suitable bound on the introduced parameter $t$.

Proposition 2.7. Assume that $\tau$ does not vanish in $[-1,1]$ and that $\left\{\pi_{i}\right\}_{i \in \mathbb{N}_{0}}$ are exceptional Legendre polynomials with

$$
\int_{-1}^{1} \frac{\pi_{i_{1}}(u) \pi_{i_{2}}(u)}{\tau(u)^{2}} \mathrm{~d} u=\nu_{i_{1}} \delta_{i_{1} i_{2}}
$$

for constants $\nu_{i}>0, i \in \mathbb{N}_{0}$ and completeness in $\mathrm{L}^{2}\left([-1,1], \tau^{-2} \mathrm{~d} z\right)$. Let $m \in \mathbb{N}_{0}$ and set

$$
\nu_{m ; i}:= \begin{cases}\nu_{i} & \text { if } i \neq m, \\ \left(t+\nu_{m}^{-1}\right)^{-1} & \text { if } i=m .\end{cases}
$$

Then, $\tau_{m}(z)>0$ on $[-1,1]$ if and only if $\nu_{m ; m}>0$. In that case, the set $\left\{\pi_{m ; i}(z ; t)\right\}_{i \in \mathbb{N}_{0}}$ is a family of exceptional Legendre polynomials with

$$
\int_{-1}^{1} \frac{\pi_{m ; i_{1}}(u) \pi_{m ; i_{2}}(u)}{\tau_{m}(u)^{2}} \mathrm{~d} u=\nu_{m ; i_{1}} \delta_{i_{1} i_{2}}, \quad i_{1}, i_{2}, m \in \mathbb{N}_{0},
$$

and completeness in $\mathrm{L}^{2}\left([-1,1], \tau_{m}^{-2} \mathrm{~d} z\right)$.

Proof. First, note that (2.10) is true in a formal sense. By (2.9), the rational function

$$
\rho_{m ; i_{1} i_{2}}(z ; t):=\rho_{i_{1} i_{2}}(z)-\frac{t \rho_{i_{1} m}(z) \rho_{i_{2} m}(z)}{1+t \rho_{m m}(z)}
$$

is defined by the integral on the l.h.s. of (2.9). Furthermore, since we are assuming that

$$
\rho_{i_{1} i_{2}}(1)=\delta_{i_{1} i_{2}} \nu_{i_{1}}
$$

we have

$$
\rho_{m ; i_{1} i_{2}}(1 ; t)=\delta_{i_{1} i_{2}}\left(\nu_{i_{1}}-\delta_{i_{1} m} \frac{t \nu_{m}^{2}}{1+t \nu_{m}}\right)=\delta_{i_{1} i_{2}} \nu_{m ; i_{1}} .
$$

By $(2.4), \tau_{m}(z)$ is positive on $z \in[-1,1]$ if and only if the same is true for $1+t \rho_{m m}(z)$. Since $\rho_{m m}(z)$ is an increasing function, the latter is true if and only if $1+t \nu_{m}>0$. Observe that

$$
\nu_{m ; m}^{-1}=t+\nu_{m}^{-1}=\nu_{m}^{-1}\left(1+t \nu_{m}\right) .
$$

Hence $\tau_{m}(z)$ is positive on $z \in[-1,1]$ if and only if $\nu_{m ; m}>0$.

Finally, we prove completeness. We assume that the eigenpolynomials $\left\{\pi_{i}(z)\right\}_{i \in \mathbb{N}_{0}}$ are $L^{2}$ complete in $[-1,1]$ relative to $\tau(z)^{-2} \mathrm{~d} z$. Following an argument adapted from the appendix of [1], we re-express the completeness assumption as

$$
\sum_{i \in \mathbb{N}_{0}} \nu_{i}^{-1} \frac{\pi_{i}(z)}{\tau(z)} \frac{\pi_{i}(w)}{\tau(w)}=\delta(z-w),
$$

where the equality is understood in distributional sense on $[-1,1] \times[-1,1]$. Rewriting $(2.3)$ as

$$
\rho_{i_{1} i_{2}}(z)=\int_{-1}^{1} \theta(z-u) \frac{\pi_{i_{1}}(u) \pi_{i_{2}}(u)}{\tau(u)^{2}} \mathrm{~d} u, \quad i_{1}, i_{2} \in \mathbb{N}_{0},
$$


where $\theta(z)$ denotes the Heaviside step function, it follows that for $j \in \mathbb{N}_{0}$

$$
\begin{aligned}
& \sum_{i \in \mathbb{N}_{0}} \nu_{i}^{-1} \frac{\pi_{i}(z)}{\tau(z)} \rho_{i j}(w)=\theta(w-z) \frac{\pi_{j}(z)}{\tau(z)}, \\
& \sum_{i \in \mathbb{N}_{0}} \nu_{i}^{-1} \rho_{i j}(z) \rho_{i j}(w)=\theta(w-z) \rho_{j j}(z)+\theta(z-w) \rho_{j j}(w) .
\end{aligned}
$$

By (2.4) and (2.5), we have

$$
\begin{aligned}
& \frac{\pi_{m ; i}(z)}{\tau_{m}(z)}=\frac{\pi_{i}(z)}{\tau(z)}-t \rho_{i m}(z) \frac{\pi_{m}(z)}{\tau_{m}(z)}, \quad i \in \mathbb{N}_{0}, \\
& \frac{t \rho_{m m}(z)}{\tau_{m}(z)}=\frac{1}{\tau(z)}-\frac{1}{\tau_{m}(z)} .
\end{aligned}
$$

Therefore, making use of the previous identities,

$$
\begin{aligned}
\sum_{i \in \mathbb{N}_{0}} \nu_{m ; i}^{-1} & \frac{\pi_{m ; i}(z)}{\tau_{m}(z)} \frac{\pi_{m ; i}(w)}{\tau_{m}(w)}=t \frac{\pi_{m ; m}(z)}{\tau_{m}(z)} \frac{\pi_{m ; m}(w)}{\tau_{m}(w)}+\sum_{i \in \mathbb{N}_{0}} \nu_{i}^{-1} \frac{\pi_{m ; i}(z)}{\tau_{m}(z)} \frac{\pi_{m ; i}(w)}{\tau_{m}(w)} \\
= & t \frac{\pi_{m}(z)}{\tau_{m}(z)} \frac{\pi_{m}(w)}{\tau_{m}(w)}+\sum_{i \in \mathbb{N}_{0}} \nu_{i}^{-1}\left(\frac{\pi_{i}(z)}{\tau(z)}-t \rho_{i m}(z) \frac{\pi_{m}(z)}{\tau_{m}(z)}\right)\left(\frac{\pi_{i}(w)}{\tau(w)}-t \rho_{i m}(w) \frac{\pi_{m}(w)}{\tau_{m}(w)}\right) \\
= & t \frac{\pi_{m}(z)}{\tau_{m}(z)} \frac{\pi_{m}(w)}{\tau_{m}(w)}+\delta(z-w)-t \theta(w-z) \frac{\pi_{m}(z)}{\tau(z)} \frac{\pi_{m}(w)}{\tau_{m}(w)}-t \theta(z-w) \frac{\pi_{m}(z)}{\tau_{m}(z)} \frac{\pi_{m}(w)}{\tau(w)} \\
& +t^{2}\left(\theta(w-z) \rho_{m m}(z)+\theta(z-w) \rho_{m m}(w)\right) \frac{\pi_{m}(z)}{\tau_{m}(z)} \frac{\pi_{m}(w)}{\tau_{m}(w)}=\delta(z-w) .
\end{aligned}
$$

\section{Recursive construction and proof of theorems}

The strategy to prove the main theorems is the following. First, we will define some polynomials and rational functions recursively, starting the recursion at the objects corresponding to the classical Legendre Sturm-Liouville problem. The recursion formulas coincide with (1.6), (2.5) and (2.9). Next, we show in Proposition 3.2 that these recursively defined objects coincide with those defined in Definition 1.4, and thus they satisfy the rationality and polynomiality conditions of Remark 2.2. Propositions 2.3-2.7 then ensure that at each step of the recursion we have an exceptional Legendre Sturm-Liouville problem, provided the parameters are chosen in the right range.

Definition 3.1. Let $i, i_{1}, i_{2} \in \mathbb{N}_{0}, \boldsymbol{m}=\boldsymbol{m}_{n}=\left(m_{1}, \ldots, m_{n}\right) \in \mathbb{N}_{0}^{n}$ and $\boldsymbol{m}_{j}=\left(m_{1}, \ldots, m_{j}\right)$, and define recursively functions $R_{\boldsymbol{m} ; i_{1} i_{2}}\left(z ; \boldsymbol{t}_{\boldsymbol{m}}\right), \tilde{\tau}_{\boldsymbol{m}}\left(z ; \boldsymbol{t}_{\boldsymbol{m}}\right)$ and $\tilde{P}_{\boldsymbol{m} ; i}\left(z ; \boldsymbol{t}_{\boldsymbol{m}}\right)$. For $j=0$, we start the recursion at $\boldsymbol{m}_{0}=\varnothing$ with

$$
\begin{aligned}
& R_{\boldsymbol{m}_{0} ; i_{1} i_{2}}\left(z ; \boldsymbol{t}_{\boldsymbol{m}_{0}}\right)=R_{i_{1} i_{2}}(z), \\
& \tilde{\tau}_{\boldsymbol{m}_{0}}\left(z ; \boldsymbol{t}_{\boldsymbol{m}_{0}}\right)=1, \\
& \tilde{P}_{\boldsymbol{m}_{0} ; i}\left(z ; \boldsymbol{t}_{\boldsymbol{m}_{0}}\right)=P_{i}(z),
\end{aligned}
$$

where $R_{i_{1} i_{2}}(z)$ are given by $(1.5)$ and $P_{i}(z)$ are the classical Legendre operators. For $j=1, \ldots, n$ we define recursively

$$
\begin{aligned}
R_{\boldsymbol{m}_{j} ; i_{1} i_{2}}\left(z ; \boldsymbol{t}_{\boldsymbol{m}_{j}}\right)= & R_{\boldsymbol{m}_{j-1} ; i_{1} i_{2}}\left(z ; \boldsymbol{t}_{\boldsymbol{m}_{j-1}}\right) \\
& -\frac{t_{m_{j}} R_{\boldsymbol{m}_{j-1} ; i_{1} m_{j}}\left(z ; \boldsymbol{t}_{\boldsymbol{m}_{j-1}}\right) R_{\boldsymbol{m}_{j-1} ; i_{2} m_{j}}\left(z ; \boldsymbol{t}_{\boldsymbol{m}_{j-1}}\right)}{1+t_{m_{j}} R_{\boldsymbol{m}_{j-1} ; m_{j} m_{j}}\left(z ; \boldsymbol{t}_{\boldsymbol{m}_{j-1}}\right)},
\end{aligned}
$$




$$
\begin{aligned}
& \tilde{\tau}_{\boldsymbol{m}_{j}}\left(z ; \boldsymbol{t}_{\boldsymbol{m}_{j}}\right)=\left(1+t_{m_{j}} R_{\boldsymbol{m}_{j-1} ; m_{j} m_{j}}\left(z ; \boldsymbol{t}_{\boldsymbol{m}_{j-1}}\right)\right) \tilde{\tau}_{\boldsymbol{m}_{j-1}}\left(z ; \boldsymbol{t}_{\boldsymbol{m}_{j-1}}\right) \\
& \tilde{P}_{\boldsymbol{m}_{j} ; i}\left(z ; \boldsymbol{t}_{\boldsymbol{m}_{j}}\right)=\left(1+t_{m_{j}} R_{\boldsymbol{m}_{j-1} ; m_{j} m_{j}}\left(z ; \boldsymbol{t}_{\boldsymbol{m}_{j-1}}\right)\right) \tilde{P}_{\boldsymbol{m}_{j-1} ; i}\left(z ; \boldsymbol{t}_{\boldsymbol{m}_{j-1}}\right) \\
&-t_{m_{j}} R_{\boldsymbol{m}_{j-1} ; m_{j}}\left(z ; \boldsymbol{t}_{\boldsymbol{m}_{j-1}}\right) \tilde{P}_{\boldsymbol{m}_{j-1} ; m_{j}}\left(z ; \boldsymbol{t}_{\boldsymbol{m}_{j-1}}\right) .
\end{aligned}
$$

The next proposition states that these recursively defined functions coincide with the polynomials and rational functions introduced in Definition 1.4.

Proposition 3.2. For $i, i_{1}, i_{2} \in \mathbb{N}_{0}$ and $\boldsymbol{m}=\left(m_{1}, \ldots, m_{n}\right) \in \mathbb{N}_{0}^{n}$, let $R_{\boldsymbol{m} ; i_{1} i_{2}}\left(z ; \boldsymbol{t}_{\boldsymbol{m}}\right), \tilde{\tau}_{\boldsymbol{m}}\left(z ; \boldsymbol{t}_{\boldsymbol{m}}\right)$ and $\tilde{P}_{\boldsymbol{m} ; i}\left(z ; \boldsymbol{t}_{\boldsymbol{m}}\right)$ be the functions defined in Definition 3.1. Let $\tau_{\boldsymbol{m}}\left(z ; \boldsymbol{t}_{\boldsymbol{m}}\right)$ and $P_{\boldsymbol{m} ; i}\left(z ; \boldsymbol{t}_{\boldsymbol{m}}\right)$ be the polynomials defined in Definition 1.4. Then,

$$
\begin{aligned}
& \tilde{\tau}_{\boldsymbol{m}}\left(z ; \boldsymbol{t}_{\boldsymbol{m}}\right)=\tau_{\boldsymbol{m}}\left(z ; \boldsymbol{t}_{\boldsymbol{m}}\right), \\
& \tilde{P}_{\boldsymbol{m} ; i}\left(z ; \boldsymbol{t}_{\boldsymbol{m}}\right)=P_{\boldsymbol{m} ; i}\left(z ; \boldsymbol{t}_{\boldsymbol{m}}\right),
\end{aligned}
$$

and

$$
R_{\boldsymbol{m} ; i_{1} i_{2}}\left(z ; \boldsymbol{t}_{\boldsymbol{m}}\right)=\int_{-1}^{z} \frac{P_{\boldsymbol{m} ; i_{1}}\left(u ; \boldsymbol{t}_{\boldsymbol{m}}\right) P_{\boldsymbol{m} ; i_{2}}\left(u ; \boldsymbol{t}_{\boldsymbol{m}}\right)}{\tau_{\boldsymbol{m}}\left(u ; \boldsymbol{t}_{\boldsymbol{m}}\right)^{2}} \mathrm{~d} u,
$$

where, again, the integral denotes an anti-derivative that vanishes at $z=-1$.

The consequence of this proposition is to ensure that the rational CDTs applied iteratively on the classical Legendre operator are always well defined, i.e., the conditions specified in Remark 2.2 will hold at each step of the chain. Before we can address the proof of Proposition 3.2, we need to establish the following technical lemma.

Lemma 3.3. Let $\boldsymbol{m} \in \mathbb{N}_{0}^{n}$ with $n \geq 3$. Then

$$
\begin{aligned}
& \left(\begin{array}{cc}
1+t_{m_{n-1}} R_{\boldsymbol{m}_{n-2} ; m_{n-1} m_{n-1}}\left(z ; \boldsymbol{t}_{\boldsymbol{m}_{n-2}}\right) & t_{m_{n}} R_{\boldsymbol{m}_{n-2} ; m_{n-1} m_{n}}\left(z ; \boldsymbol{t}_{\boldsymbol{m}_{n-2}}\right) \\
t_{m_{n-1}} R_{\boldsymbol{m}_{n-2} ; m_{n-1} m_{n}}\left(z ; \boldsymbol{t}_{\boldsymbol{m}_{n-2}}\right) & 1+t_{m_{n}} R_{\boldsymbol{m}_{n-2} ; m_{n} m_{n} ;\left(z ; \boldsymbol{t}_{\boldsymbol{m}_{n-2}}\right)}
\end{array}\right)^{-1} \\
& \quad=\mathcal{M}_{n-1, n}\left(\mathcal{R}_{\boldsymbol{m}}\left(z ; t_{\boldsymbol{m}}\right)^{-1}\right)
\end{aligned}
$$

where $\mathcal{M}_{n-1, n}$ denotes the bottom right $2 \times 2$ submatrix of the indicated matrix and $\mathcal{R}_{\boldsymbol{m}}\left(z ; t_{\boldsymbol{m}}\right)$ is given in Definition 1.4.

Proof. The result follows by iteration and the Sylvester determinant identity [2]. Let us start by showing the argument for $n=3$. If we write

$$
\mathcal{R}_{\boldsymbol{m}}\left(z ; \boldsymbol{t}_{\boldsymbol{m}}\right)=\left(\begin{array}{c|c}
a & \boldsymbol{b}_{1} \\
\hline \boldsymbol{b}_{2}^{\mathrm{T}} & \mathcal{A}
\end{array}\right),
$$

where $\boldsymbol{b}_{j}$ are 2 -tuples for $j=1,2$ and $\mathcal{A}$ is the $\mathcal{M}_{2,3}$ submatrix, by the Sylvester determinant identity,

$$
\mathcal{M}_{2,3}\left(\mathcal{R}_{\boldsymbol{m}}\left(z ; \boldsymbol{t}_{\boldsymbol{m}}\right)^{-1}\right)=\left(\mathcal{A}-\boldsymbol{b}_{2}^{\mathrm{T}} a^{-1} \boldsymbol{b}_{1}\right)^{-1} .
$$

Identifying the elements in the decomposition of $\mathcal{R}_{\boldsymbol{m}}\left(z ; \boldsymbol{t}_{\boldsymbol{m}}\right)$ according to its definition, by direct calculation and the recursive formulation (3.1) we obtain

$$
\mathcal{A}-\boldsymbol{b}_{1} a^{-1} \boldsymbol{b}_{2}^{\mathrm{T}}=\left(\begin{array}{cc}
1+t_{m_{2}} R_{m_{1} ; m_{2} m_{2}}\left(z ; t_{m_{1}}\right) & t_{m_{3}} R_{m_{1} ; m_{2} m_{3}}\left(z ; t_{m_{1}}\right) \\
t_{m_{2}} R_{m_{1} ; m_{2} m_{3}}\left(z ; t_{m_{1}}\right) & 1+t_{m_{3}} R_{m_{1} ; m_{3} m_{3}}\left(z ; t_{m_{1}}\right)
\end{array}\right)
$$

as desired. 
For $n>3$, let $\mathcal{R}_{\boldsymbol{m}}^{[j]}\left(z ; \boldsymbol{t}_{\boldsymbol{m}}\right)$ be the $(n-j) \times(n-j)$ matrix for any $j \in\{1, n-2\}$ with entries

$$
\left[\mathcal{R}_{\boldsymbol{m}}^{[j]}\left(z ; \boldsymbol{t}_{\boldsymbol{m}}\right)\right]_{k \ell}:=\delta_{k \ell}+t_{m_{j+\ell}} R_{\boldsymbol{m}_{j} ; m_{j+k} m_{j+\ell}}\left(z ; \boldsymbol{t}_{\boldsymbol{m}_{j}}\right), \quad k, \ell \in\{1, \ldots, n-j\} .
$$

We aim to show that $\mathcal{R}_{\boldsymbol{m}}^{[n-2]}\left(z ; \boldsymbol{t}_{\boldsymbol{m}}\right)^{-1}=\mathcal{M}_{n-1, n}\left(\mathcal{R}_{\boldsymbol{m}}\left(z ; t_{\boldsymbol{m}}\right)^{-1}\right)$. Arguing as above, we have $\mathcal{R}_{\boldsymbol{m}}^{[n-2]}\left(z ; \boldsymbol{t}_{\boldsymbol{m}}\right)^{-1}=\mathcal{M}_{2,3}\left(\mathcal{R}_{\boldsymbol{m}}^{[n-3]}\left(z ; t_{\boldsymbol{m}}\right)^{-1}\right)$. Applying analogously the Sylvester determinant identity, we also obtain

$$
\mathcal{R}_{\boldsymbol{m}}^{[j+1]}\left(z ; \boldsymbol{t}_{\boldsymbol{m}}\right)^{-1}=\mathcal{M}_{2, n-j}\left(\mathcal{R}_{\boldsymbol{m}}^{[j]}\left(z ; t_{\boldsymbol{m}}\right)^{-1}\right)
$$

for $j \in\{0, \ldots, n-3\}$. The result then follows by iteration.

Proof of Proposition 3.2. Throughout this proof, we are going to omit the explicit dependence on $z$ and $\boldsymbol{t}_{\boldsymbol{m}}$ of the objects, which must be understood from the dependence on $\boldsymbol{m}$, i.e., we will write $\mathcal{R}_{\boldsymbol{m}}$ instead of $\mathcal{R}_{\boldsymbol{m}}\left(z ; \boldsymbol{t}_{\boldsymbol{m}}\right)$. By definition, we have

$$
\mathcal{R}_{\boldsymbol{m}}=\left(\begin{array}{c|c}
\mathcal{R}_{\boldsymbol{m}_{n-1}} & \vdots \\
\hline \cdots & 1+t_{m_{n}} R_{m_{n} m_{n}}
\end{array}\right) .
$$

Applying the expression for the inverse with the adjoint of the cofactors matrix, we obtain that

$$
\left[\mathcal{R}_{\boldsymbol{m}}^{-1}\right]_{n n}=\frac{\operatorname{det} \mathcal{R}_{\boldsymbol{m}_{n-1}}}{\operatorname{det} \mathcal{R}_{\boldsymbol{m}_{n}}}=\frac{\tau_{\boldsymbol{m}_{n-1}}}{\tau_{\boldsymbol{m}_{n}}} .
$$

Applying Lemma 3.3 after computing the inverse of the matrix in the left hand side of (3.7) and using the recursion (3.1) allows to prove that the $\tau_{\boldsymbol{m}}$ and $\tau_{\boldsymbol{m}_{n-1}}$ satisfy the recursion relation (3.2). Since $\tilde{\tau}_{\boldsymbol{m}_{1}}=\tau_{\boldsymbol{m}_{1}}$, we see that (3.4) holds.

In order to prove (3.5), we first observe that (3.3) can be rewritten as

$$
\tilde{P}_{\boldsymbol{m} ; i}=\frac{\tau_{(\boldsymbol{m} ; i)}}{\tau_{\boldsymbol{m}_{n-1}}}\left[\left(\mathcal{R}_{(\boldsymbol{m}, i)}^{[n-1]}\right)^{-1}\left(\tilde{P}_{\boldsymbol{m}_{n-1} ; m_{n}}, \tilde{P}_{\boldsymbol{m}_{n-1} ; i}\right)^{\mathrm{T}}\right]_{1},
$$

where $\mathcal{R}_{(\boldsymbol{m}, i)}^{[n-1]}=\mathcal{R}_{(\boldsymbol{m}, i)}^{[n-1]}\left(z ; \boldsymbol{t}_{(\boldsymbol{m}, i)}\right)$ is given by (3.8). The proof follows by induction. It is clear that (3.5) holds for $\boldsymbol{m}_{1}$ by definition. We assume that it also holds for $\boldsymbol{m}_{j}$ with $j=1, \ldots, n-1$, and we must prove it also hods for $\boldsymbol{m}_{n}$. We start by proving that,

$$
\left(\mathcal{R}_{(\boldsymbol{m}, i)}^{[n-1]}\right)^{-1}\left(\begin{array}{c}
P_{\boldsymbol{m}_{n-1} ; m_{n}} \\
P_{\boldsymbol{m}_{n-1} ; i}
\end{array}\right)=\frac{\tau_{\boldsymbol{m}_{n-1}}}{\tau_{\boldsymbol{m}_{n-2}}}\left(\left(\mathcal{R}_{(\boldsymbol{m}, i)}^{[n-2]}\right)^{-1}\left(\begin{array}{c}
P_{\boldsymbol{m}_{n-2} ; m_{n-1}} \\
P_{\boldsymbol{m}_{n-2} ; m_{n}} \\
P_{\boldsymbol{m}_{n-2} ; i}
\end{array}\right)\right)^{\langle 1\rangle},
$$

where in the right hand side we have the last two components of a vector of three entries. This may be verified as follows. First note that, by assumption, we have $\tilde{P}_{\boldsymbol{m}_{j} ; i}=P_{\boldsymbol{m}_{j} ; i}$ for all $i \in \mathbb{N}_{0}$ and $j=1, \ldots, n-1$. By Lemma 3.3 and (3.3), the left hand side is equal to

$$
\mathcal{M}_{2,3}\left(\left(\mathcal{R}_{(\boldsymbol{m}, i)}^{[n-2]}\right)^{-1}\right)\left(\begin{array}{ccc}
-t_{m_{n-1}} R_{\boldsymbol{m}_{n-2} ; m_{n-1}, m_{n}} & \frac{\tau_{\boldsymbol{m}_{n-1}}}{\tau_{\boldsymbol{m}_{n-2}}} & 0 \\
\left.-t_{m_{n-1}} R_{\boldsymbol{m}_{n-2} ; m_{n-1}, i}\right) & 0 & \frac{\tau_{\boldsymbol{m}_{n-1}}}{\tau_{\boldsymbol{m}_{n-2}}}
\end{array}\right)\left(\begin{array}{c}
P_{\boldsymbol{m}_{n-2} ; m_{n-1}} \\
P_{\boldsymbol{m}_{n-2} ; m_{n}} \\
P_{\boldsymbol{m}_{n-2} ; i}
\end{array}\right),
$$

where we have used by $(3.2)$

$$
1+t_{m_{n-1}} R_{\boldsymbol{m}_{n-2} ; m_{n-1}, m_{n-1}}=\frac{\tau_{\boldsymbol{m}_{n-1}}}{\tau_{\boldsymbol{m}_{n-2}}} .
$$


Now we can conclude that

$$
\mathcal{M}_{2,3}\left(\left(\mathcal{R}_{(\boldsymbol{m}, i)}^{[n-2]}\right)^{-1}\right)\left(\begin{array}{ccc}
-t_{m_{n-1}} R_{\boldsymbol{m}_{n-2} ; m_{n-1}, m_{n}} & \frac{\tau_{\boldsymbol{m}_{n-1}}}{\tau_{\boldsymbol{m}_{n-2}}} & 0 \\
-t_{m_{n-1}} R_{\boldsymbol{m}_{n-2} ; m_{n-1}, i} & 0 & \frac{\tau_{\boldsymbol{m}_{n-1}}}{\tau_{\boldsymbol{m}_{n-2}}}
\end{array}\right)
$$

corresponds to the last two rows of $\left(\mathcal{R}_{(\boldsymbol{m}, i)}^{[n-2]}\right)^{-1}$ multiplied by $\frac{\tau_{\boldsymbol{m}_{n-1}}}{\tau_{\boldsymbol{m}_{n-2}}}$. For the second block, corresponding to $\mathcal{M}_{2,3}\left(\left(\mathcal{R}_{(\boldsymbol{m}, i)}^{[n-2]}\right)^{-1}\right)$, the correspondence is clear. In order to verify the result for the elements $(2,1)$ and $(3,1)$ of $\left(\mathcal{R}_{(\boldsymbol{m}, i)}^{[n-2]}\right)^{-1}$, we identify the components of the second matrix in (3.10) as elements of $\mathcal{R}_{(\boldsymbol{m}, i)}^{[n-2]}$ and we rely on the fact that we are multiplying elements of the matrix $\mathcal{R}_{(\boldsymbol{m}, i)}^{[n-2]}$ and its inverse. In fact, for instance the element $(2,1)$ corresponds to

$$
\begin{gathered}
-\left[\left(\mathcal{R}_{(\boldsymbol{m}, i)}^{n-2}\right)^{-1}\right]_{2,2}\left[\mathcal{R}_{(\boldsymbol{m}, i)}^{[n-2]}\right]_{2,1}-\left[\left(\mathcal{R}_{(\boldsymbol{m}, i)}^{[n-2]}\right)^{-1}\right]_{2,3}\left[\mathcal{R}_{(\boldsymbol{m}, i)}^{[n-2]}\right]_{3,1} \\
=-[\mathcal{I}]_{2,1}+\left[\left(\mathcal{R}_{(\boldsymbol{m}, i)}^{[n-2]}\right)^{-1}\right]_{2,1}\left[\mathcal{R}_{(\boldsymbol{m}, i)}^{[n-2]}\right]_{1,1} \\
=\left(1+t_{m_{n-1}} R_{\boldsymbol{m}_{n-2} ; m_{n-1} m_{n-1}}\right)\left[\left(\mathcal{R}_{(\boldsymbol{m}, i)}^{[n-2]}\right)^{-1}\right]_{2,1}=\frac{\tau_{\boldsymbol{m}_{n-1}}}{\tau_{\boldsymbol{m}_{n-2}}}\left[\left(\mathcal{R}_{(\boldsymbol{m}, i)}^{[n-2]}\right)^{-1}\right]_{2,1},
\end{gathered}
$$

where $\mathcal{I}$ is the identity matrix. Similarly to (3.9), we can show the following identity for $j=1, \ldots, n-2$ :

$$
\left(\mathcal{R}_{(\boldsymbol{m}, i)}^{[j]}\right)^{-1}\left(\begin{array}{c}
P_{\boldsymbol{m}_{j} ; m_{j+1}} \\
\vdots \\
P_{\boldsymbol{m}_{j} ; m_{n}} \\
\boldsymbol{P}_{\boldsymbol{m}_{j} ; i}
\end{array}\right)=\frac{\tau_{\boldsymbol{m}_{j}}}{\tau_{\boldsymbol{m}_{j-1}}}\left(\left(\mathcal{R}_{(\boldsymbol{m}, i)}^{[j-1]}\right)^{-1}\left(\begin{array}{c}
P_{\boldsymbol{m}_{j-1} ; m_{j}} \\
\vdots \\
P_{\boldsymbol{m}_{j-1} ; m_{n}} \\
P_{\boldsymbol{m}_{j-1} ; i}
\end{array}\right)\right)^{\langle 1\rangle} .
$$

Combining the previous identities yields (3.5):

$$
\begin{aligned}
\tilde{P}_{\boldsymbol{m} ; i} & =\frac{\tau_{(\boldsymbol{m} ; i)}}{\tau_{\boldsymbol{m}_{n-1}}}\left[\left(\mathcal{R}_{(\boldsymbol{m}, i)}^{[n-1]}\right)^{-1}\left(\tilde{P}_{\boldsymbol{m}_{n-1} ; m_{n}}, \tilde{P}_{\boldsymbol{m}_{n-1} ; i}\right)^{\mathrm{T}}\right]_{1}, \\
& =\frac{\tau_{(\boldsymbol{m} ; i)}}{\tau_{\boldsymbol{m}_{n-2}}}\left[\left(\mathcal{R}_{(\boldsymbol{m}, i)}^{[n-2]}\right)^{-1}\left(P_{\boldsymbol{m}_{n-2} ; m_{n-1}}, P_{\boldsymbol{m}_{n-2} ; m_{n}}, P_{\boldsymbol{m}_{n-2} ; i}\right)^{\mathrm{T}}\right]_{1}=\cdots \\
& =\tau_{(\boldsymbol{m} ; i)}\left[\left(\mathcal{R}_{(\boldsymbol{m}, i)}\right)^{-1}\left(P_{m_{1}}, \ldots P_{m_{n}}, P_{i}\right)^{\mathrm{T}}\right]_{1}=P_{\boldsymbol{m} ; i}
\end{aligned}
$$

Finally, relation (3.6) follows by Proposition 2.6 and by induction on $j$.

Proposition 3.2 together with the results in Section 2 allow now to prove the main theorems.

Proof of Theorems 1.5 and 1.6. The key to the proof is to observe that starting form the classical Legendre operator, the application of a rational confluent Darboux transformation indexed by an integer $m_{i}$ introduces an extra real parameter $t_{m_{i}}$ and leads to a well defined Sturm-Liouville problem defining a family of exceptional Legendre polynomials. Indeed, the equivalence of the objects defined by the CDT recursion (2.3)-(2.5) and those defined by matrix multiplication in Definition 1.4 show that for any $\boldsymbol{m} \in \mathbb{N}_{0}^{n}, \tau_{\boldsymbol{m}}\left(z ; \boldsymbol{t}_{\boldsymbol{m}}\right)$ and $P_{\boldsymbol{m} ; i}\left(z ; \boldsymbol{t}_{\boldsymbol{m}}\right)$ are polynomials and $\mathcal{R}_{\boldsymbol{m}}\left(z ; \boldsymbol{t}_{\boldsymbol{m}}\right)$ is a matrix of rational functions that satisfy the premises of Propositions 2.3-2.7 (see Remark 2.2). Theorem 1.5 follows then from Proposition 2.5 and Theorem 1.6 follows by induction from Proposition 2.7, which establishes the bounds on the parameters (1.10) that ensure the regularity of $T_{\boldsymbol{m}}\left(\boldsymbol{t}_{\boldsymbol{m}}\right)$ and the positivity of the measure in $[-1,1]$ 
As mentioned above, the degree of the $i$-th exceptional Legendre polynomial $P_{\boldsymbol{m} ; i}$ indexed by $\boldsymbol{m}=\left(m_{1}, \ldots, m_{n}\right) \in \mathbb{N}_{0}^{n}$ is not $i$. The next proposition provides this result.

Proposition 3.4. Let $\boldsymbol{m}=\left(m_{1}, \ldots, m_{n}\right)$ and suppose that $m_{1}, \ldots, m_{n}$ are distinct. Let $\tau_{\boldsymbol{m}}, P_{\boldsymbol{m} ; i}$ be as defined in (1.6) and (1.8). Then,

$$
\begin{aligned}
\operatorname{deg} \tau_{\boldsymbol{m}}\left(z ; \boldsymbol{t}_{\boldsymbol{m}}\right)= & 2\left(m_{1}+\ldots+m_{n}\right)+n, \\
\operatorname{deg} P_{\boldsymbol{m} ; i}\left(z ; \boldsymbol{t}_{\boldsymbol{m}}\right)= & 2\left(m_{1}+\ldots+m_{n}\right)+n+i \\
& -\left(\delta_{i, m_{1}}+\cdots+\delta_{i, m_{n}}\right)(2 i+1), \quad i \in \mathbb{N}_{0} .
\end{aligned}
$$

Moreover,

$$
P_{\boldsymbol{m} ; m_{k}}\left(z ; \boldsymbol{t}_{\boldsymbol{m}}\right)=P_{\boldsymbol{m}^{\langle k\rangle} ; m_{k}}\left(z ; \boldsymbol{t}_{\boldsymbol{m}\langle k\rangle}\right), \quad k=1, \ldots, n,
$$

where $\boldsymbol{m}^{\langle k\rangle} \in \mathbb{N}_{0}^{n-1}$ denotes the tuple obtained by removing the $k^{\text {th }}$ entry of $\boldsymbol{m}$.

Notice that (3.13) accounts for the above Kronecker delta terms in (3.12). It is also worth noting that, as opposed to the "traditional" exceptional families, the degree sequence for exceptional Legendre polynomials is not an increasing sequence, which is further evidence of the different construction.

Proof. The identity (3.13) follows directly from applying (3.3) to this specific choice and the symmetry with respect to permutations in $\boldsymbol{m}$.

Relations (3.11) and (3.12) can be proved by induction. It is clear that they hold for $\boldsymbol{m}_{1}=m_{1}$, since $\operatorname{deg} R_{i_{1} i_{2}}(z)=i_{1}+i_{2}+1$. Notice that in $P_{m_{1} ; i}\left(z ; t_{m_{1}}\right)$, the coefficients of $R_{m_{1} m_{1}}(z) P_{i}(z)$ and $R_{m_{1} i}(z) P_{m_{1}}(z)$ do not coincide if $i \neq m_{1}$, so $\operatorname{deg} P_{m_{1} ; i}\left(z ; t_{m_{1}}\right)=2 m_{1}+i+1$. If $i=m_{1}$, by $(3.13)$ we obtain $\operatorname{deg} P_{m_{1} ; m_{1}}\left(z ; t_{m_{1}}\right)=\operatorname{deg} P_{m_{1}}(z)=m_{1}$

Now we assume (3.11) and (3.12) hold for $\boldsymbol{m}_{j-1}$. By (3.6), the degree of $R_{\boldsymbol{m}_{j-1} ; m_{j}}\left(z ; \boldsymbol{t}_{\boldsymbol{m}_{j-1}}\right)$, understood as the difference between the degree of the polynomial numerator and of the polynomial denominator, is $m_{j}+i+1$ if $i \neq m_{1}, \ldots, m_{j-1}$. Then,

$$
\operatorname{deg} \tau_{\boldsymbol{m}_{j}}\left(z ; \boldsymbol{t}_{\boldsymbol{m}_{j}}\right)=\operatorname{deg} \tau_{\boldsymbol{m}_{j-1}}\left(z ; \boldsymbol{t}_{\boldsymbol{m}_{j-1}}\right)+2 m_{j}+1=2\left(m_{1}+\cdots+m_{j}\right)+j .
$$

Arguing as above to verify that there is no cancellation between the highest order contributions, we obtain

$$
\operatorname{deg} P_{\boldsymbol{m}_{j} ; i}\left(z ; \boldsymbol{t}_{\boldsymbol{m}_{j}}\right)=2\left(m_{1}+\cdots+m_{j-1}\right)+(j-1)+2 m_{j}+i+1
$$

if $i \neq m_{1}, \ldots, m_{j}$. In order to prove the result for $i$ equal to some component of $\boldsymbol{m}_{j}$, we employ relation (3.13).

Remark 3.5. From Proposition 3.4 we see that the codimension (number of missing degrees) of the exceptional Legendre family indexed by $\boldsymbol{m}=\left(m_{1}, \ldots, n\right)$ is $2\left(m_{1}+\cdots+m_{n}\right)+n$. This coincides with the degree of $\tau_{\boldsymbol{m}}$, as it happens for all exceptional polynomials [7].

So far we have considered the case when $\boldsymbol{m}=\left(m_{1}, \ldots, m_{n}\right)$ contains distinct indices. As announced in Remark (1.7), let us show that this choice entails no loss of generality.

Proposition 3.6. Let $\boldsymbol{m} \in \mathbb{N}_{0}^{n}$ and let $\tau_{\boldsymbol{m}}\left(z ; \boldsymbol{t}_{\boldsymbol{m}}\right)$ and $P_{\boldsymbol{m} ; i}\left(z ; \boldsymbol{t}_{\boldsymbol{m}}\right)$ be as defined in (1.6) and (1.8). Then, for any $j \in \mathbb{N}_{0}$ we have

$$
\begin{aligned}
& \tau_{(\boldsymbol{m}, j, j)}\left(z ;\left(\boldsymbol{t}_{\boldsymbol{m}}, t_{j}, t_{j}\right)\right)=\tau_{(\boldsymbol{m}, j)}\left(z ;\left(\boldsymbol{t}_{\boldsymbol{m}}, 2 t_{j}\right)\right), \\
& P_{(\boldsymbol{m}, j, j) ; i}\left(z ;\left(\boldsymbol{t}_{\boldsymbol{m}}, t_{j}, t_{j}\right)\right)=P_{(\boldsymbol{m}, j) ; i}\left(z ;\left(\boldsymbol{t}_{\boldsymbol{m}}, 2 t_{j}\right)\right) .
\end{aligned}
$$


We see thus that the repeated application of a 2-step confluent Darboux transformation at the same eigenvalue only serves to modify the deformation parameter. In general, if the two parameters at the repeated $j$ are different, we would have similarly

$$
\begin{aligned}
& \tau_{(\boldsymbol{m}, j, j)}\left(z ;\left(\boldsymbol{t}_{\boldsymbol{m}}, t_{j}, t_{j}^{\prime}\right)=\tau_{(\boldsymbol{m}, j)}\left(z ;\left(\boldsymbol{t}_{\boldsymbol{m}}, t_{j}+t_{j}^{\prime}\right)\right)\right), \\
& P_{(\boldsymbol{m}, j, j) ; i}\left(z ;\left(\boldsymbol{t}_{\boldsymbol{m}}, t_{j}, t_{j}^{\prime}\right)\right)=P_{(\boldsymbol{m}, j) ; i}\left(z ;\left(\boldsymbol{t}_{\boldsymbol{m}}, t_{j}+t_{j}^{\prime}\right) .\right.
\end{aligned}
$$

Proof. We omit again explicit dependence on $z$ and $t_{\boldsymbol{m}}, t_{j}$ if no confusion arises. We apply Proposition 3.2 and Definition 3.1 twice to obtain

$$
\begin{aligned}
\tau_{(\boldsymbol{m}, j, j)}= & \left(1+t_{j} R_{(\boldsymbol{m}, j) ; j j}\right) \tau_{(\boldsymbol{m}, j)}=\left(1+t_{j} R_{\boldsymbol{m} ; j j}-\frac{t_{j}^{2} R_{\boldsymbol{m} ; j j}^{2}}{1+t_{j} R_{\boldsymbol{m} ; j j}}\right)\left(1+t_{j} R_{\boldsymbol{m} ; j j}\right) \tau_{\boldsymbol{m}} \\
= & \left(1+2 t_{j} R_{\boldsymbol{m} ; j j}\right) \tau_{\boldsymbol{m}}=\tau_{(\boldsymbol{m}, j)}\left(\left(z ;\left(\boldsymbol{t}_{\boldsymbol{m}}, 2 t_{j}\right)\right),\right. \\
P_{(\boldsymbol{m}, j, j) ; i}= & \left(1+t_{j} R_{(\boldsymbol{m}, j) ; j j}\right) P_{(\boldsymbol{m}, j) ; i}-t_{j} R_{(\boldsymbol{m}, j) ; i j} P_{(\boldsymbol{m}, j) ; j} \\
= & \left(1+t_{j} R_{(\boldsymbol{m}, j) ; j j}\right)\left(\left(1+t_{j} R_{\boldsymbol{m} ; j j}\right) P_{\boldsymbol{m} ; i}-t_{j} R_{\boldsymbol{m} ; i j} P_{\boldsymbol{m} ; i}\right) \\
& -t_{j}\left(R_{\boldsymbol{m} ; i j}-\frac{t_{j} R_{\boldsymbol{m} ; j j} R_{\boldsymbol{m} ; i j}}{1+t_{j} R_{\boldsymbol{m} ; j j}}\right) P_{\boldsymbol{m} ; j} \\
= & \left(1+2 t_{j} R_{\boldsymbol{m} ; j j}\right) P_{\boldsymbol{m} ; i}-2 t_{j} R_{\boldsymbol{m} ; i j} P_{\boldsymbol{m} ; j}=P_{(\boldsymbol{m}, j) ; i}\left(z ;\left(\boldsymbol{t}_{\boldsymbol{m}}, 2 t_{j}\right)\right) .
\end{aligned}
$$

\section{Examples}

To conclude, we present some examples of exceptional Legendre polynomials and orthogonality relations for the cases of $n=1$ and $n=2$.

\subsection{The 1-parameter exceptional Legendre family}

In the 1-parameter case, we have $\boldsymbol{m}=\left(m_{1}\right) \in \mathbb{N}_{0}$, and

$$
\begin{aligned}
& \tau_{m_{1}}\left(z ; t_{m_{1}}\right)=1+t_{m_{1}} R_{m_{1} m_{1}}(z), \\
& P_{m_{1} ; i}\left(z ; t_{m_{1}}\right)=\left(1+t_{m_{1}} R_{m_{1} m_{1}}(z)\right) P_{i}(z)-t_{m_{1}} R_{i m_{1}}(z) P_{m_{1}}(z), \quad i \in \mathbb{N}_{0} .
\end{aligned}
$$

Note that $P_{m_{1} ; m_{1}}(z)=P_{m_{1}}(z)$. The degrees of the other polynomials are

$$
\operatorname{deg} P_{m_{1} ; i}(z)=i+2 m_{1}+1, \quad i \neq m_{1} .
$$

The corresponding exceptional operator is $T_{m_{1}}\left(t_{m_{1}}\right)=\hat{T}\left(\tau_{m_{1}}\left(z ; t_{m_{1}}\right)\right)$ with the latter as per (1.1).

The polynomial $\tau_{m_{1}}\left(z ; t_{m_{1}}\right)$ does not vanish in $[-1,1]$ provided that

$$
t_{m_{1}}>-\frac{1}{R_{m_{1} m_{1}}(1)}=-m_{1}-\frac{1}{2} .
$$

In this case, $\left\{P_{m_{1} ; i}\left(z ; t_{m_{1}}\right)\right\}_{i \in \mathbb{N}_{0}}$ is a family of exceptional Legendre polynomials, with orthogonality weight

$$
W_{m_{1}}\left(z ; t_{m_{1}}\right)=\frac{1}{\tau_{m_{1}}\left(z ; t_{m_{1}}\right)^{2}} .
$$

The above set is a complete orthogonal polynomial basis of the space $\mathrm{L}^{2}\left([-1,1], W_{m_{1}}\left(z ; t_{m_{1}}\right) \mathrm{d} z\right)$. The orthogonality relations are

$$
\begin{aligned}
& \int_{-1}^{1} \frac{P_{m_{1} ; i_{1}}\left(u ; t_{m_{1}}\right) P_{m_{1} ; i_{2}}\left(u ; t_{m_{1}}\right)}{\tau_{m_{1}}\left(u ; t_{m_{1}}\right)^{2}} \mathrm{~d} u=\frac{2}{1+2 i_{1}} \delta_{i_{1} i_{2}}, \quad i_{1}, i_{2} \in \mathbb{N}_{0} \backslash\left\{m_{1}\right\} \\
& \int_{-1}^{1} \frac{P_{m_{1} ; m_{1}}\left(u ; t_{m_{1}}\right)^{2}}{\tau_{m_{1}}\left(u ; t_{m_{1}}\right)^{2}} \mathrm{~d} u=\frac{2}{1+2 m_{1}+2 t_{m_{1}}} .
\end{aligned}
$$




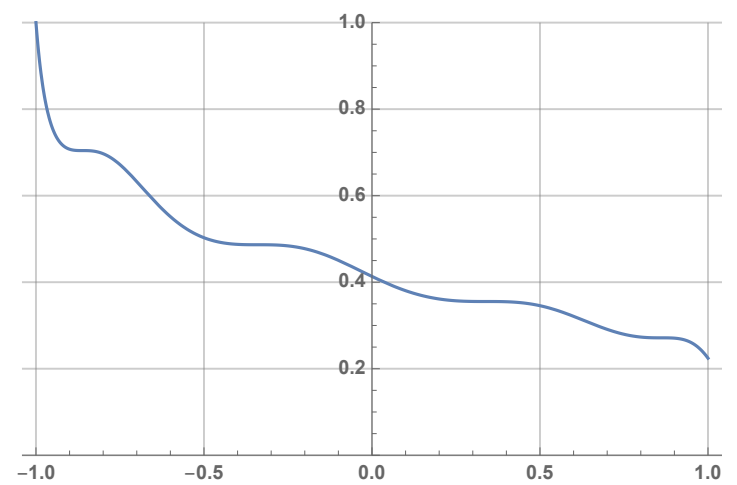

Figure 1. Exceptional Legendre weight $\tau_{m_{1}}^{-2}\left(z, t_{m_{1}}\right)$ for $m_{1}=4$ and $t_{m_{1}}=5.2$.

The above example illustrates perfectly the isospectral nature of the CDT that relates $T(1) \rightarrow$ $T_{m_{1}}\left(t_{m_{1}}\right)$. The eigenvalues of the two operators are the same. As for the eigenfunctions, if $t_{m_{1}} \neq 0$, then for $i \neq m_{1}$ they are transformed, but their norms stay the same. On the other hand, for $i=m_{1}$ the opposite happens: the eigenfunction does not change but its norm does.

Since $\tau_{m_{1}}^{\prime}(z)=t_{m_{1}} P_{m_{1}}^{2}(z)$, it follows that the weight $W_{m_{1}}(z)$ is a decreasing (resp. increasing) function for $t_{m_{1}}>0$ (resp. $\left.t_{m_{1}}<0\right)$, which has $m_{1}$ saddle points in $[-1,1]$ at the zeros of $P_{m_{1}}$, but no local minima or maxima.

For instance, for $m_{1}=4$, we have

$$
\tau_{4}\left(z, t_{4}\right)=1+\frac{1}{576} t_{4}\left(64+81 z-540 z^{3}+1998 z^{5}-2700 z^{7}+1225 z^{9}\right)
$$

and the weight is shown in Fig. 1. The first few polynomials for this choice are

$$
\begin{aligned}
& P_{4 ; 0}\left(z, t_{4}\right)=1+\frac{t_{4}}{144}\left(16+135 z^{3}-459 z^{5}+585 z^{7}-245 z^{9}\right), \\
& P_{4 ; 1}\left(z, t_{4}\right)=z+\frac{t_{4}}{1152}\left(-9+128 z+171 z^{2}+30 z^{4}-1314 z^{6}+2475 z^{8}-1225 z^{10}\right), \\
& P_{4 ; 2}\left(z, t_{4}\right)=P_{2}(z)-\frac{t_{4}}{576}\left(32-96 z^{2}+189 z^{3}-756 z^{5}+1278 z^{7}-1300 z^{9}+525 z^{11}\right), \\
& P_{4 ; 3}\left(z, t_{4}\right)=P_{3}(z)-\frac{t_{4}}{9216}\left(243-1536 z-3402 z^{2}+2560 z^{3}+3645 z^{4}+7668 z^{6}-17955 z^{8}\right. \\
& \left.\quad+16950 z^{10}-6125 z^{12}\right), \\
& \left.P_{4 ; 4}\left(z, t_{4}\right)=P_{4}(z), \quad-42255 z^{8}+66171 z^{10}-50855 z^{12}+15435 z^{14}\right) . \\
& P_{4 ; 5}\left(z, t_{4}\right)=P_{5}(z)+\frac{t_{4}}{9216}\left(243+1920 z-1215 z^{2}-8960 z^{3}-3645 z^{4}+8064 z^{5}+17145 z^{6}\right.
\end{aligned}
$$

We display the above polynomials for $t_{4}=0$ (classical Legendre) and for $t_{4}=5.2$ in Fig. 2 . Observe that all polynomials undergo a continuous deformation as $t_{4}$ changes, except for $P_{4 ; 4}=P_{4}$ that stays the same.

\subsection{The 2-parameter exceptional Legendre family}

In the 2-parameter case, we have $\boldsymbol{m}=\left(m_{1}, m_{2}\right) \in \mathbb{N}_{0}^{2}, \boldsymbol{t}_{\boldsymbol{m}}=\left(t_{m_{1}}, t_{m_{2}}\right)$ and

$$
\begin{aligned}
\tau_{\boldsymbol{m}}\left(z ; \boldsymbol{t}_{\boldsymbol{m}}\right)= & \tau_{m_{1}}\left(z ; t_{m_{1}}\right) \tau_{m_{2}}\left(z ; t_{m_{2}}\right)-t_{m_{1}} t_{m_{2}} R_{m_{1} m_{2}}(z)^{2}, \\
P_{\boldsymbol{m} ; i}\left(z ; \boldsymbol{t}_{\boldsymbol{m}}\right)= & P_{i}(z) \tau_{\boldsymbol{m}}\left(z ; \boldsymbol{t}_{\boldsymbol{m}}\right)-P_{m_{1}}(z) t_{m_{1}} \tau_{m_{2}}\left(z ; t_{m_{2}}\right) R_{m_{1} ; m_{2}, i}\left(z ; t_{m_{2}}\right) \\
& -P_{m_{2}}(z) t_{m_{2}} \tau_{m_{1}}\left(z ; t_{m_{1}}\right) R_{m_{1} ; m_{2}, i}\left(z ; t_{m_{1}}\right), \quad i \in \mathbb{N}_{0},
\end{aligned}
$$



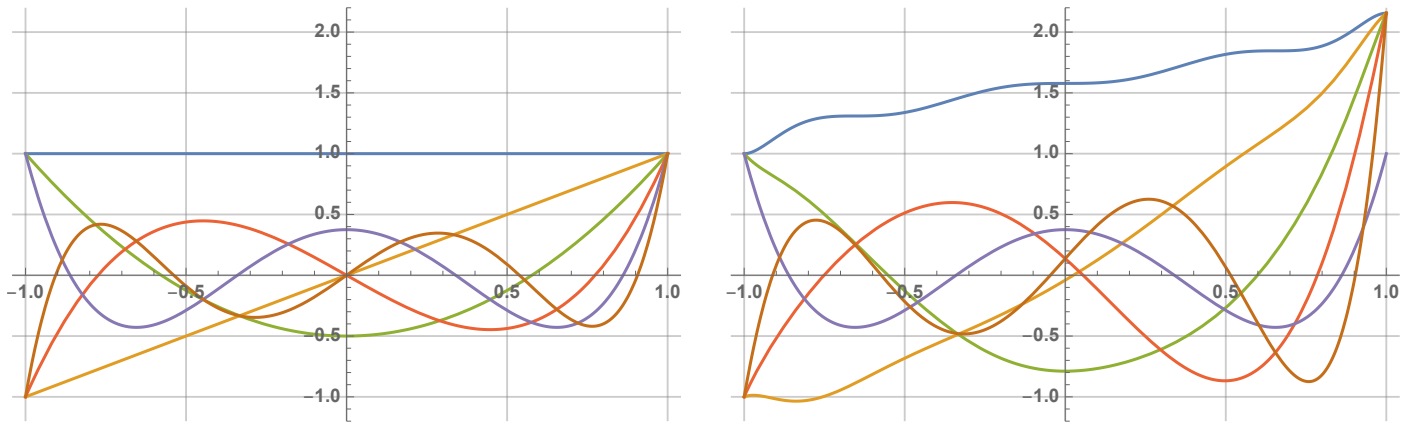

Figure 2. First few exceptional Legendre polynomials $P_{m_{1} ; i}\left(z ; t_{m_{1}}\right)$ for $m_{1}=4$, with $t_{m_{1}}=0$ (left) and $t_{m_{1}}=5.2$ (right).

where

$$
\begin{aligned}
R_{m_{1} ; i_{1} i_{2}}\left(z ; t_{m}\right) & =\int_{-1}^{z} \frac{P_{m_{1} ; i_{1}}\left(u ; t_{m_{1}}\right) P_{m_{1} ; i_{2}}\left(u ; t_{m_{1}}\right)}{\tau_{m_{1}}\left(u ; t_{m_{1}}\right)^{2}} \mathrm{~d} u \\
& =R_{i_{1} i_{2}}(z)-\frac{t_{m_{1}} R_{i_{1} m_{1}}(z) R_{i_{2} m_{1}}(z)}{1+t_{m_{1}} R_{m_{1} m_{1}}}, \quad i_{1}, i_{2} \in \mathbb{N}_{0},
\end{aligned}
$$

Supposing that $m_{1} \neq m_{2}$ we have

$$
\begin{aligned}
& \operatorname{deg} P_{\boldsymbol{m} ; i}\left(z ; \boldsymbol{t}_{\boldsymbol{m}}\right)=i+2 m_{1}+2 m_{2}+2, \quad i \in \mathbb{N}_{0} \backslash\left\{m_{1}, m_{2}\right\}, \\
& \operatorname{deg} P_{\boldsymbol{m}, m_{1}}\left(z ; \boldsymbol{t}_{\boldsymbol{m}}\right)=m_{1}+2 m_{2}+1, \\
& \operatorname{deg} P_{\boldsymbol{m} ; m_{2}}\left(z ; \boldsymbol{t}_{\boldsymbol{m}}\right)=m_{2}+2 m_{1}+1 .
\end{aligned}
$$

The polynomial $\tau_{\boldsymbol{m}}\left(z ; t_{m_{1}, t_{m_{2}}}\right)$ does not vanish in $[-1,1]$ provided that

$$
t_{m_{1}}>-m_{1}-\frac{1}{2}, \quad t_{m_{2}}>-m_{2}-\frac{1}{2} .
$$

In this case, $\left\{P_{\boldsymbol{m} ; i}\left(z ; \boldsymbol{t}_{\boldsymbol{m}}\right)\right\}_{i \in \mathbb{N}_{0}}$ is a family of exceptional Legendre polynomials, with orthogonality weight

$$
W_{\boldsymbol{m}}\left(z ; \boldsymbol{t}_{\boldsymbol{m}}\right)=\frac{1}{\tau_{\boldsymbol{m}}\left(z ; \boldsymbol{t}_{\boldsymbol{m}}\right)^{2}} .
$$

The above set is a complete orthogonal polynomial basis of the $\operatorname{space~}^{2}\left([-1,1], W_{\boldsymbol{m}}\left(z ; \boldsymbol{t}_{\boldsymbol{m}}\right) \mathrm{d} z\right)$.

The orthogonality relations are

$$
\begin{aligned}
& \int_{-1}^{1} \frac{P_{\boldsymbol{m} ; i_{2}}\left(u ; t_{\boldsymbol{m}}\right) P_{\boldsymbol{m} ; i_{2}}\left(u ; \boldsymbol{t}_{\boldsymbol{m}}\right)}{\tau_{\boldsymbol{m}}\left(u ; \boldsymbol{t}_{\boldsymbol{m}}\right)^{2}} \mathrm{~d} u=\frac{2}{1+2 i_{1}} \delta_{i_{1} i_{2}}, \quad i_{1}, i_{2} \in \mathbb{N}_{0} \backslash\left\{m_{1}, m_{2}\right\}, \\
& \int_{-1}^{1} \frac{P_{\boldsymbol{m} ; m_{1}}\left(u ; \boldsymbol{t}_{\boldsymbol{m}}\right)^{2}}{\tau_{\boldsymbol{m}}\left(u ; \boldsymbol{t}_{\boldsymbol{m}}\right)^{2}} \mathrm{~d} u=\frac{2}{1+2 m_{1}+2 t_{m_{1}}} \\
& \int_{-1}^{1} \frac{P_{\boldsymbol{m} ; m_{2}}\left(u ; \boldsymbol{t}_{\boldsymbol{m}}\right)^{2}}{\tau_{\boldsymbol{m}}\left(u ; \boldsymbol{t}_{\boldsymbol{m}}\right)^{2}} \mathrm{~d} u=\frac{2}{1+2 m_{2}+2 t_{m_{2}}} .
\end{aligned}
$$

For instance, for $\boldsymbol{m}=\left(m_{1}, m_{1}\right)=(1,2)$, we have

$$
\begin{aligned}
\tau_{(1,2)}\left(z ;\left(t_{1}, t_{2}\right)\right)= & 1+\frac{1}{3} t_{1}\left(1+z^{3}\right)+\frac{1}{20} t_{2}\left(4+5 z-10 z^{3}+9 z^{5}\right) \\
& +\frac{1}{960} t_{1} t_{2}(1+z)^{4}\left(49-116 z+110 z^{2}-36 z^{3}+9 z^{4}\right) .
\end{aligned}
$$

For certain values of $\boldsymbol{t}_{\boldsymbol{m}}=\left(t_{m_{1}}, t_{m_{2}}\right)$ the weight is displayed in Fig. 3. To the best of our knowledge, this is the first example of an exceptional orthogonal polynomial system whose weight is not monotonic or unimodal. 


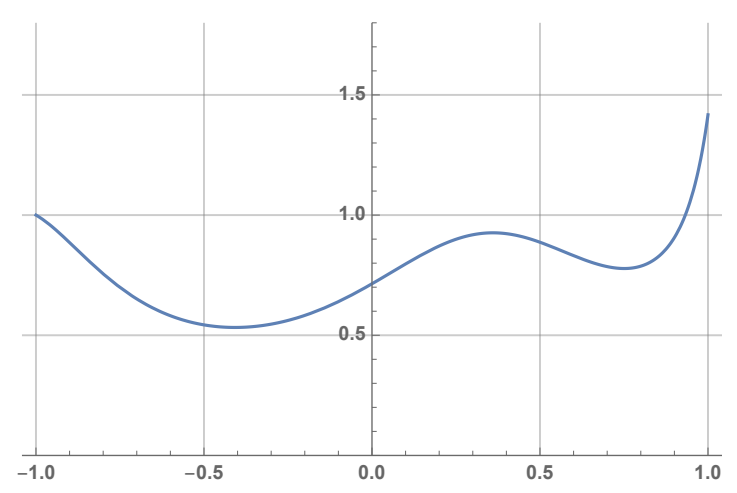

Figure 3. Exceptional Legendre weight $\tau_{\boldsymbol{m}}^{-2}\left(z, \boldsymbol{t}_{\boldsymbol{m}}\right)$ for $\boldsymbol{m}=\left(m_{1}, m_{2}\right)=(1,2)$ and $\left(t_{m_{1}}, t_{m_{2}}\right)=(2,-1.6)$.

\section{Summary}

In this paper we show that the class of exceptional orthogonal polynomials is much larger than previously thought. A new construction based on confluent Darboux transformations leads to new exceptional families with some similarities and differences with respect to the other exceptional families. In common, they are also Sturm-Liouville problems with rational coefficients, each family is indexed by a set of integers, and it defines a complete basis of polynomial eigenfunctions, whose degree sequence has missing degrees. But as opposed to the other exceptional families, there are no gaps in the spectrum and the new families contain an arbitrary number of real deformation parameters, so the construction can be seen as an isospectral deformation of the classical operators. We illustrate the new construction by describing the full class of exceptional Legendre polynomials, which cannot be derived through the standard construction. The same method can be applied with minor modifications to the Jacobi operator. A more exhaustive description of these matters will be provided in a forthcoming publication, together with a discussion of the implications for the classification of exceptional polynomials [8].

\section{Acknowledgements}

MAGF would like to thank the Max-Planck-Institute for Mathematics in the Sciences, Leipzig (Germany), where some of her work took place. DGU acknowledges support from the Spanish MICINN under grants PGC2018-096504-B-C33 and RTI2018-100754-B-I00 and the European Union under the 2014-2020 ERDF Operational Programme and by the Department of Economy, Knowledge, Business and University of the Regional Government of Andalusia (project FEDERUCA18-108393).

\section{References}

[1] Abraham P.B., Moses H.E., Changes in potentials due to changes in the point spectrum: anharmonic oscillators with exact solutions, Phys. Rev. A 22 (1980), 1333-1340.

[2] Akritas A.G., Akritas E.K., Malaschonok G.I., Various proofs of Sylvester's (determinant) identity, Math. Comput. Simulation 42 (1996), 585-593.

[3] Bonneux N., Exceptional Jacobi polynomials, J. Approx. Theory 239 (2019), 72-112, arXiv:1804.01323.

[4] Contreras-Astorga A., Schulze-Halberg A., Recursive representation of Wronskians in confluent supersymmetric quantum mechanics, J. Phys. A: Math. Theor. 50 (2017), 105301, 15 pages, arXiv:1702.00843.

[5] Durán A.J., Higher order recurrence relation for exceptional Charlier, Meixner, Hermite and Laguerre orthogonal polynomials, Integral Transforms Spec. Funct. 26 (2015), 357-376, arXiv:1409.4697.

[6] Durán A.J., Exceptional Hahn and Jacobi orthogonal polynomials, J. Approx. Theory 214 (2017), 9-48, arXiv:1510.02579. 
[7] García-Ferrero M.A., Gómez-Ullate D., Milson R., A Bochner type characterization theorem for exceptional orthogonal polynomials, J. Math. Anal. Appl. 472 (2019), 584-626, arXiv:1603.04358.

[8] García-Ferrero M.A., Gómez-Ullate D., Milson R., Confluent Darboux transformations and exceptional orthogonal polynomials, in preparation.

[9] Gesztesy F., Teschl G., On the double commutation method, Proc. Amer. Math. Soc. 124 (1996), 1831-1840.

[10] Gómez-Ullate D., Grandati Y., Milson R., Rational extensions of the quantum harmonic oscillator and exceptional Hermite polynomials, J. Phys. A: Math. Theor. 47 (2014), 015203, 27 pages, arXiv:1306.5143.

[11] Gómez-Ullate D., Kamran N., Milson R., An extended class of orthogonal polynomials defined by a SturmLiouville problem, J. Math. Anal. Appl. 359 (2009), 352-367, arXiv:0807.3939.

[12] Gómez-Ullate D., Kamran N., Milson R., A conjecture on exceptional orthogonal polynomials, Found. Comput. Math. 13 (2013), 615-666, arXiv:1203.6857.

[13] Gómez-Ullate D., Kasman A., Kuijlaars A.B.J., Milson R., Recurrence relations for exceptional Hermite polynomials, J. Approx. Theory 204 (2016), 1-16, arXiv:1506.03651.

[14] Gómez-Ullate D., Marcellán F., Milson R., Asymptotic and interlacing properties of zeros of exceptional Jacobi and Laguerre polynomials, J. Math. Anal. Appl. 399 (2013), 480-495, arXiv:1204.2282.

[15] Grandati Y., Bérard A., Comments on the generalized SUSY QM partnership for Darboux-Pöschl-Teller potential and exceptional Jacobi polynomials, J. Engrg. Math. 82 (2013), 161-171.

[16] Grandati Y., Quesne C., Confluent chains of DBT: enlarged shape invariance and new orthogonal polynomials, SIGMA 11 (2015), 061, 26 pages, arXiv:1503.07747.

[17] Hemery A.D., Veselov A.P., Whittaker-Hill equation and semifinite-gap Schrödinger operators, J. Math. Phys. 51 (2010), 072108, 17 pages, arXiv:0906.1697.

[18] Horváth Á.P., The electrostatic properties of zeros of exceptional Laguerre and Jacobi polynomials and stable interpolation, J. Approx. Theory 194 (2015), 87-107, arXiv:1410.0906.

[19] Keung W.-Y., Sukhatme U.P., Wang Q.M., Imbo T.D., Families of strictly isospectral potentials, J. Phys. A: Math. Gen. 22 (1989), L987-L992.

[20] Kuijlaars A.B.J., Milson R., Zeros of exceptional Hermite polynomials, J. Approx. Theory 200 (2015), 28-39, arXiv:1412.6364.

[21] Marquette I., Quesne C., New families of superintegrable systems from Hermite and Laguerre exceptional orthogonal polynomials, J. Math. Phys. 54 (2013), 042102, 16 pages, arXiv:1211.2957.

[22] Miki H., Tsujimoto S., A new recurrence formula for generic exceptional orthogonal polynomials, J. Math. Phys. 56 (2015), 033502, 13 pages, arXiv:1410.0183.

[23] Odake S., Recurrence relations of the multi-indexed orthogonal polynomials. III, J. Math. Phys. 57 (2016), 023514, 24 pages, arXiv:1509.08213.

[24] Odake S., Sasaki R., Infinitely many shape invariant potentials and new orthogonal polynomials, Phys. Lett. B 679 (2009), 414-417, arXiv:0906.0142.

[25] Post S., Tsujimoto S., Vinet L., Families of superintegrable Hamiltonians constructed from exceptional polynomials, J. Phys. A: Math. Theor. 45 (2012), 405202, 10 pages, arXiv:1206.0480.

[26] Quesne C., Solvable rational potentials and exceptional orthogonal polynomials in supersymmetric quantum mechanics, SIGMA 5 (2009), 084, 24 pages, arXiv:0906.2331.

[27] Schulze-Halberg A., Roy B., Darboux partners of pseudoscalar Dirac potentials associated with exceptional orthogonal polynomials, Ann. Physics 349 (2014), 159-170, arXiv:1409.0999.

[28] Sparenberg J.M., Baye D., Supersymmetric transformations of real potentials on the line, J. Phys. A: Math. Gen. 28 (1995), 5079-5095.

[29] Sukumar C.V., Supersymmetric quantum mechanics and the inverse scattering method, J. Phys. A: Math. Gen. 18 (1985), 2937-2955.

[30] Szegő G., Orthogonal Polynomials, American Mathematical Society Colloquium Publications, Vol. 23, Amer. Math. Soc., New York, 1939. 